Elsevier required licence: (C) <2019>. This manuscript version is made available under the CC-BY-NCND 4.0 license http://creativecommons.org/licenses/by-nc-nd/4.0/

The definitive publisher version is available online at

[https://www.sciencedirect.com/science/article/pii/S0169260718317693?via\%3Dihub] 


\title{
Half a century of Computer Methods and Programs in Biomedicine: A bibliometric analysis from 1970 - 2017
}

\author{
Nagesh Shukla ${ }^{1}$, José M. Merigó ${ }^{1,2}$, Thorsten Lammers ${ }^{1}$, Luis Miranda ${ }^{2}$

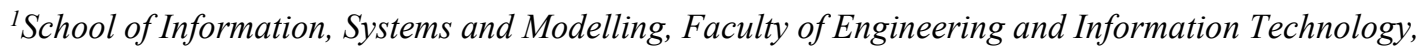 \\ University of Technology Sydney, 81 Broadway, Ultimo 2007, NSW, Australia \\ ${ }^{2}$ Department of Management Control and Information Systems, School of Economics and Business, \\ University of Chile, Av. Diagonal Paraguay 257, 8330015 Santiago, Chile \\ Emails:Nagesh.Shukla@uts.edu.au; Jose.Merigo@uts.edu.au; Thorsten.Lammers@uts.edu.au
}

\begin{abstract}
Background and Objective:

Computer Methods and Programs in Biomedicine (CMPB) is a leading international journal that presents developments about computing methods and their application in biomedical research. The journal published its first issue in 1970. In 2020, the journal celebrates the 50th anniversary. Motivated by this event, this article presents a bibliometric analysis of the publications of the journal during this period $(1970-2017)$.

Methods:

The objective is to identify the leading trends occurring in the journal by analysing the most cited papers, keywords, authors, institutions and countries. For doing so, the study uses the Web of Science Core Collection database. Additionally, the work presents a graphical mapping of the bibliographic information by using the visualization of similarities (VOS) viewer software. This is done to analyze bibliographic coupling, co-citation and co-occurrence of keywords.
\end{abstract}

Results:

$\mathrm{CMPB}$ is identified as a leading and core journal for biomedical researchers. The journal is strongly connected to IEEE Transactions on Biomedical Engineering and IEEE Transactions on Medical Imaging. Paper from Wang, Jacques, Zheng (published in 1995) is its most cited document. The top author in this journal is James Geoffrey Chase and the top contributing institution is Uppsala U (Sweden). Most of the papers in CMPB are from the USA followed by the UK and Italy. China and Taiwan are the only Asian countries to appear in the top 10 publishing in CMPB. A keyword co-occurrences analysis revealed strong co-occurrences for classification, picture archiving and communication system (PACS), heart rate variability, survival analysis and simulation. Keywords analysis for the last decade revealed that machine learning for a variety of healthcare problems (including image processing and analysis) dominated other research fields in CMPB.

Conclusions:

It can be concluded that CMPB is a world-renowned publication outlet for biomedical researchers which has been growing in a number of publications since 1970. The analysis also conclude that the journal is very 
international with publications from all over the world although today European countries are the most productive ones.

Keywords: Bibliometrics; Web of Science; Co-citation; VOS viewer.

\section{Introduction}

Computer Methods and Programs in Biomedicine (CMPB) is one of the main journals in the field of biomedical research which focuses on the development of formal computing methods and their application in biomedical research and practice. The journal started publishing papers from 1970 under the name Computer Programs in Biomedicine. In 1985, it was renamed to Computer Methods and Programs in Biomedicine. Prof Werner Schneider from Uppsala University was its first managing editor in 1970. Since then, the journal has been publishing theoretical and application oriented papers addressing biomedical research challenges and problems. In 2013, Editor-in-Chief of the journal was changed to Prof YuChuan Li. The journal initially started publishing less than 4 issues a year, later increasing to 6 issues and after 2015, it has grown to publish more than 12 issues per year suggesting a growing interest of the research community in the journal. CMPB is considered to be an A* journal in the field of Biomedical Engineering in Australia (according to 2010 Excellence in Research Australia John Lamp journal ranking). Currently, its CiteScore is 3.49 and the impact factor is 2.674. It is in top $20 \%$ of journals in Computer Science Applications, Software and Health Informatics discipline.

To celebrate CMPB's $50^{\text {th }}$ anniversary, this paper develops a bibliometric analysis of the leading trends observed in the biomedical research field through this journal from 1970 to 2017. It is important to keep track of topic changes and other bibliometric trends to maximise the benefits of the use of computing methods in medical and biomedical research fields so that novel and innovative applications of computational methods can be embraced. As the topics of research change over time, it is difficult to track and analyse them appropriately. The bibliographic research employed in this study aims to provide an overview of topic trends and challenges dealt with in the literature. Bibliometric analysis is applied to the literature published in CMPB since its inception. We also analyse the dynamic trend of topics covered in CMPB over time. In the analysis, leading topics, authors, institutions and countries along with citation structure and analysis are identified and discussed. In this work, we have 
employed the Web of Science (WoS) Core Collection database to collect and analyse the bibliographic material. We used the visualization of similarities (VOS) viewer software developed in Van Eck \& Waltman [1] to systematically visualise the bibliographic data. The visualisation work presented in this paper used range of analysis including bibliographic coupling [2], co-citation [3], citation, co-authorship and co-occurrence of keywords.

When keywords of the papers published in CMPB were analysed, it was revealed that research related to classification, PACS (picture archiving and communication system), heart rate variability, survival analysis and simulation were popular in the journal since its inception. The analysis also revealed the popular themes of research in the CMPB publications. The most popular themes were: (i) machine learning methods for medical data analysis, (ii) signal processing in case of heart rate variability, (iii) simulation models for diabetes, (iv) use of PACS data for its use in health technology assessments and image processing, (v) decision support systems development based on healthcare records and (vi) survivability analysis for health conditions. It is common in the literature to develop some sort of activities such as editorials, reviews and special issues for the journal to mark a significant anniversary. Many journals recently have presented a bibliometric analysis of their publications by celebrating anniversaries. This type of approach has been used for journals including the Journal of Business Research, Knowledge-Based Systems , the Journal of Business \& Industrial Marketing, International Journal of Intelligent Systems, and the Computers in Biology and Medicine.

The rest of the paper is organised as follows. Section 2 briefly describes the bibliometric methods used in this paper for analysis. Section 3 presents the results obtained from the bibliometric analysis including the publication and citation structure, the leading authors, institutions and countries, the most cited papers and the most citing papers. Section 4 visualises the results from co-citation, bibliographic coupling of institutions, countries, and keywords. Finally, Section 5 summarizes the main findings of the presented bibliometric analysis.

\section{Methods}

Bibliometrics is a research area of library and information sciences that studies the bibliographic material by using quantitative approaches [12-13]. Due to the development of 
computers and internet, today it is quite easy to develop a bibliometric analysis since most of the information is available online [14]. Therefore, many authors have developed bibliometric studies of a wide range of areas including management [15], economics [16], innovation [17], entrepreneurship [18], fuzzy research [19] and linguistic decision making [20].

Bibliometrics is developed for a wide range of purposes including the analysis of a topic [21], journal [22], country [17] or university [23]. The analysis of a journal is of particular interest when the journal celebrates a special event like a significant anniversary. At this time, it becomes of great interest to develop a bibliometric overview of the journal in order to identify and commemorate the leading trends of the journal [24]. Many journals have already published a bibliometric analysis of their publications including the Accounting Review [25], Journal of Financial Economics [26], Strategic Management Journal [27], Technovation [28] and the Journal of Product Innovation Management [29]. Recently, many other journals are also publishing this approach including Computers \& Human Behavior [30], European Journal of Operational Research [31], Computers \& Industrial Engineering [32], Information Sciences [33], European Journal of Marketing [34], IEEE Transactions on Fuzzy Systems [35] and the Journal of Knowledge Management [36].

When analysing the bibliographic data in order to generate bibliometric results, it is important to define the bibliometric indicators to be used in the analysis in order to obtain the most representative information [37-39]. This work considers the number of papers as a measure of productivity and the number of citations as a measure of popularity and influence. Additionally, it also considers the citations per paper ratio, citations thresholds and the $h$ index [40]. The $h$-index is a measure that connects publications with citations by analysing the $X$ number of papers that have received $X$ citation or more [41-42]. The $h$-index has been extended and generalised under a wide range of frameworks [43]. In some specific cases, the study also considers some other indicators in order to get a more general picture of the results. For universities, the paper considers the general university rankings (Academic Ranking of World Universities (ARWU) and Quacquarelli \& Symonds (QS) University Ranking) in order to analyse the general standing of the institutions that publish in the journal. 
Considering the factor "countries", the work normalizes the results per million inhabitants in order to compare countries with different size in population.

In order to provide a deeper analysis of the bibliometric information, the paper develops a graphical mapping of the bibliographic data with the aim of identifying how the leading actors of the journal connect with each other. This work uses the VOS viewer [1] software. However, note that in the literature there are other software available to build graphical networks of the data $[44,47]$. VOS viewer collects the bibliographic information from a database (e.g. Web of Science or Scopus) and builds graphical maps by using bibliographic coupling, co-citation and co-occurrence of keywords [45-46]. The bibliographic coupling analyses those documents that cite the same third material [2]. Co-citation focuses on those publications that receive citations from the same third documents [3]. Co-occurrence of keywords measures those keywords that appear more frequently in the same publications.

The search of the bibliographic information was carried out in October 2018. The search used in the Web of Science Core Collection database uses the two names the journal has had between 1970 and 2018: "Computer Methods and Programs in Biomedicine" and "Computer Programs in Biomedicine". Up to December 2017, the research has found 4225 documents published in CMPB considering only articles, reviews, letters and notes. In October 2018, these documents had received 59,094 citations. The $h$-index is 82 . That is, 82 documents have received 82 citations or more.

\section{Results}

\subsection{Publication and citation structure of CMPB}

In 1970, CMPB started publishing just over 20 papers a year and an upward trend continued exceeding 50 papers annually in the nineties, until 2004, when over 100 papers a year were being published. After 2012, more than 200 papers per year have been published in the journal. Figure 1 illustrates the number of papers published in CMPB since its inception in 1970. It can be seen that the number of papers published in CMPB has increased tenfold in

the last 50 years showing growing interest of the researchers towards this journal as well as a rapid evolution of the biomedical research field in the last two decades due to growth in 
medical data/information storage, retrieval and analysis technologies and advancement of computing methods.

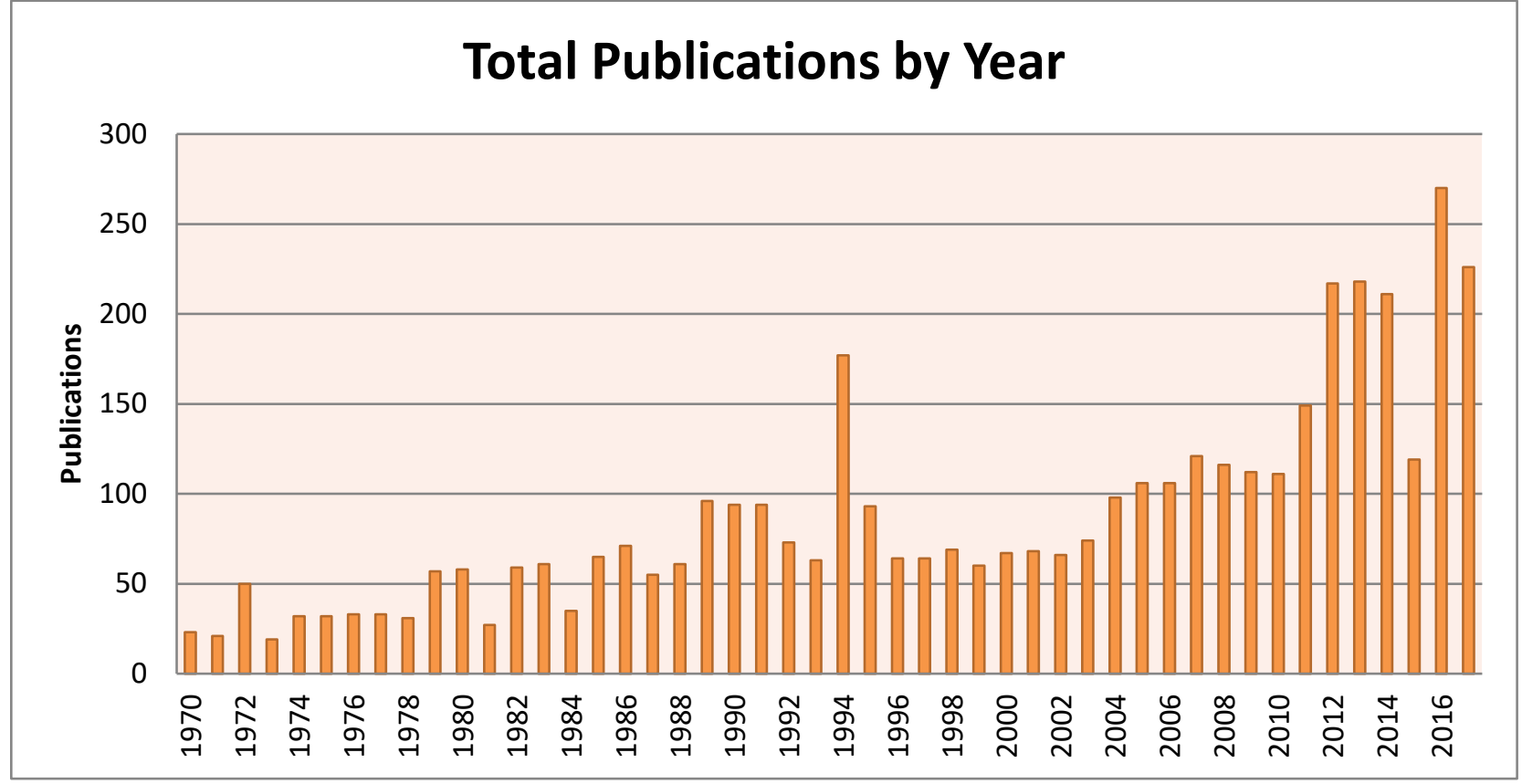

Figure 1. Annual number of papers published in CMPB

Table 1 shows the annual citation structure of the papers published in CMPB. Several citation thresholds ( uch as $\geq 200, \geq 100, \geq 50, \geq 20, \geq 10, \geq 5$, and $\geq 1$ ) have been used to study the citations arising from the papers. Table 1 indicates that CMPB has been able to attract citations by publishing papers which are highly cited. Papers published in the last few years still need some time to catch up in terms of attracting citations. CMPB has been getting high citations consistently with 31 papers $(0.73 \%)$ receiving more than 200 citations. Nearly, $14.75 \%$ of papers received more than 20 citations which is quite significant for an area not particularly amenable to high citations. It can also be seen that most of the highly cited papers are published after 1995 through to 2012 indicating improved quality of research over time.

Table 1. Annual citation structure of CMPB

\begin{tabular}{cccccccccc}
\hline Year & TP & TC & $\geq 200$ & $\geq 100$ & $\geq 50$ & $\geq 20$ & $\geq 10$ & $\geq 5$ & $\geq 1$ \\
\hline 1970 & 23 & 122 & 0 & 0 & 0 & 2 & 2 & 7 & 14 \\
1971 & 21 & 150 & 0 & 0 & 0 & 2 & 6 & 8 & 14 \\
1972 & 50 & 1096 & 1 & 1 & 2 & 5 & 9 & 16 & 24
\end{tabular}




\begin{tabular}{|c|c|c|c|c|c|c|c|c|c|}
\hline 1973 & 19 & 185 & 0 & 0 & 1 & 4 & 5 & 6 & 14 \\
\hline 1974 & 32 & 198 & 0 & 0 & 0 & 2 & 6 & 14 & 22 \\
\hline 1975 & 32 & 198 & 0 & 0 & 0 & 2 & 6 & 14 & 22 \\
\hline 1976 & 33 & 392 & 0 & 1 & 1 & 6 & 8 & 17 & 25 \\
\hline 1977 & 33 & 210 & 0 & 0 & 0 & 2 & 8 & 13 & 19 \\
\hline 1978 & 31 & 186 & 0 & 0 & 0 & 1 & 6 & 12 & 24 \\
\hline 1979 & 57 & 1587 & 2 & 3 & 5 & 7 & 12 & 22 & 45 \\
\hline 1980 & 58 & 503 & 0 & 2 & 2 & 3 & 4 & 13 & 34 \\
\hline 1981 & 27 & 376 & 0 & 1 & 1 & 3 & 8 & 11 & 19 \\
\hline 1982 & 59 & 481 & 0 & 0 & 1 & 6 & 12 & 21 & 41 \\
\hline 1983 & 61 & 1889 & 2 & 2 & 3 & 6 & 12 & 17 & 42 \\
\hline 1984 & 35 & 209 & 0 & 0 & 0 & 1 & 5 & 12 & 24 \\
\hline 1985 & 65 & 944 & 1 & 1 & 4 & 11 & 19 & 32 & 51 \\
\hline 1986 & 71 & 1255 & 2 & 2 & 2 & 8 & 18 & 29 & 50 \\
\hline 1987 & 55 & 247 & 0 & 0 & 0 & 1 & 7 & 17 & 37 \\
\hline 1988 & 61 & 473 & 0 & 0 & 2 & 6 & 11 & 18 & 37 \\
\hline 1989 & 96 & 1189 & 1 & 3 & 3 & 8 & 22 & 35 & 69 \\
\hline 1990 & 94 & 857 & 0 & 0 & 1 & 11 & 28 & 43 & 71 \\
\hline 1991 & 94 & 696 & 0 & 0 & 2 & 7 & 21 & 35 & 76 \\
\hline 1992 & 73 & 553 & 0 & 0 & 2 & 7 & 14 & 23 & 46 \\
\hline 1993 & 63 & 544 & 0 & 0 & 2 & 7 & 14 & 27 & 45 \\
\hline 1994 & 177 & 1134 & 0 & 0 & 4 & 11 & 28 & 52 & 116 \\
\hline 1995 & 93 & 2989 & 2 & 2 & 4 & 13 & 17 & 26 & 63 \\
\hline 1996 & 64 & 1208 & 1 & 2 & 4 & 12 & 20 & 38 & 55 \\
\hline 1997 & 64 & 1550 & 2 & 4 & 8 & 18 & 28 & 42 & 53 \\
\hline 1998 & 69 & 743 & 0 & 0 & 1 & 9 & 21 & 37 & 59 \\
\hline 1999 & 60 & 1982 & 3 & 4 & 5 & 18 & 28 & 42 & 56 \\
\hline 2000 & 67 & 1130 & 0 & 2 & 5 & 13 & 27 & 44 & 62 \\
\hline 2001 & 68 & 1311 & 1 & 3 & 5 & 18 & 31 & 38 & 60 \\
\hline 2002 & 66 & 1432 & 1 & 2 & 8 & 18 & 32 & 46 & 57 \\
\hline 2003 & 74 & 1149 & 0 & 0 & 4 & 16 & 32 & 48 & 64 \\
\hline 2004 & 98 & 2271 & 3 & 3 & 7 & 23 & 45 & 63 & 91 \\
\hline 2005 & 106 & 2659 & 2 & 7 & 8 & 23 & 51 & 70 & 100 \\
\hline 2006 & 106 & 2466 & 1 & 2 & 8 & 28 & 57 & 79 & 98 \\
\hline 2007 & 121 & 2087 & 0 & 2 & 7 & 34 & 53 & 80 & 110 \\
\hline 2008 & 116 & 2370 & 2 & 2 & 6 & 35 & 62 & 86 & 108 \\
\hline 2009 & 112 & 1985 & 0 & 1 & 9 & 28 & 54 & 79 & 106 \\
\hline 2010 & 111 & 2639 & 2 & 4 & 5 & 35 & 53 & 74 & 104 \\
\hline 2011 & 149 & 2157 & 0 & 2 & 8 & 29 & 53 & 90 & 133 \\
\hline 2012 & 217 & 3106 & 1 & 1 & 8 & 39 & 90 & 144 & 199 \\
\hline 2013 & 218 & 2234 & 0 & 0 & 2 & 27 & 77 & 131 & 198 \\
\hline 2014 & 211 & 2922 & 1 & 1 & 9 & 35 & 74 & 127 & 189 \\
\hline 2015 & 119 & 893 & 0 & 0 & 0 & 10 & 24 & 53 & 108 \\
\hline 2016 & 270 & 1551 & 0 & 0 & 0 & 13 & 38 & 95 & 200 \\
\hline 2017 & 226 & 586 & 0 & 0 & 0 & 0 & 7 & 31 & 115 \\
\hline Total & 4225 & 59094 & 31 & 60 & 159 & 623 & 1265 & 2077 & 3369 \\
\hline$\%$ & $100 \%$ & & $0.73 \%$ & $1.42 \%$ & $3.76 \%$ & $14.75 \%$ & $29.94 \%$ & $49.16 \%$ & $79.74 \%$ \\
\hline
\end{tabular}

Abbreviations: TP and TC $=$ Total papers and citations; $\geq 100, \geq 50, \geq 25, \geq 10, \geq 5, \geq 1=$ Number of papers with equal or more than $100,50,25,10,5$ and 1 citations.

\subsection{Influential papers in CMPB}


In terms of most cited papers published in CMPB, Table 2 presents the top 50 highly cited papers of all time. The most cited paper is from Lihong Wang, Steven L. Jacques, Liqiong Zheng published in 1995 on Monte Carlo model of steady-state light transport in multilayered tissues receiving more than 2000 citations. The next highly cited paper is from Grant A. McPherson on computer-based approach to the analysis of radioligand binding experiments published in 1983. It should be noted that most of the highly cited papers developed some type of computer programs to aid medical problems/decision making. These papers made the source codes for their programs openly available for other researchers/practitioners to use. The top most contributing authors to the highly cited papers are Natarajan Kannathal, Niclas E. Jonsson and U Rajendra Acharya each with 3 papers.

Another interesting analysis is presented in Table 3 where a list of the top 40 papers cited in CMPB papers is highlighted. The top most cited paper by Goldberger presented an opensource data archive, analysis tools and knowledge sharing forum for analysis of wellcharacterised digital recordings of physiological signals for its use by the biomedical research community. The second most cited document was the seminal paper by Cox in 1972 which proposed hazard functions and age-specific failure rates. Vast applicability of hazard functions in survival models and health risk assessments has led to the paper's popularity in CMPB. It is evident from the remaining papers in this list that machine learning based papers developing new algorithms or methods (for image analysis and numeric data analysis) were popular among the authors publishing in CMPB.

\subsection{Leading authors, institutions and countries}

In this section, a set of analyses was conducted to identify top contributing authors for CMPB. Table 4 lists all-time top authors in CMPB and their affiliations. Top authors include $Y u$ Chuan Li, James Geoffrey Chase and Geoffrey M. Shaw. The top contributing authors are from a range of institutions from Asia, US and Europe. Interesting to see that 3 authors out of top 5 leading authors are from New Zealand. These authors are also very well cited in literature as shown in Table 4. Table 5 shows the temporal evolution of top contributing authors in CMPB.

Table 2. The 50 most cited documents in CMPB

\begin{tabular}{l|l|l|l|l|l}
\hline $\mathrm{R}$ & $\mathrm{TC}$ & Title & Citations \\
per year
\end{tabular}




\begin{tabular}{|c|c|c|c|c|c|}
\hline 1 & 2083 & MCML - monte-carlo modeling of light transport in multilayered tissues & $\begin{array}{l}\text { WANG, LH; JACQUES, SL; ZHENG, } \\
\text { LQ }\end{array}$ & 1995 & 86.79 \\
\hline 2 & 1000 & A practical computer-based approach to the analysis of radioligand binding experiments & MCPHERSON, GA & 1983 & 27.78 \\
\hline 3 & 682 & DtiStudio: Resource program for diffusion tensor computation and fiber bundle tracking & $\begin{array}{l}\text { Jiang, HY; van Zijl, PCM; Kim, J; } \\
\text { Pearlson, GD; Mori, S }\end{array}$ & 2006 & 52.46 \\
\hline 4 & 654 & $\begin{array}{l}\text { Xpose - an S-PLUS based population pharmacokinetic/pharmacodynamic model building } \\
\text { aid for NONMEM }\end{array}$ & Jonsson, NE; Karlsson, MO & 1999 & 32.7 \\
\hline 5 & 549 & $\begin{array}{l}\text { MINMOD - a computer-program to calculate insulin sensitivity and pancreatic } \\
\text { responsivity from the frequently sampled intravenous glucose-tolerance test }\end{array}$ & PACINI, G; BERGMAN, RN & 1986 & 16.64 \\
\hline 6 & 533 & Program package for simulation and parameter-estimation in pharmacokinetic systems & DARGENIO, DZ; SCHUMITZKY, A & 1979 & 13.33 \\
\hline 7 & 488 & $\begin{array}{l}\text { PsN-Toolkit - A collection of computer intensive statistical methods for non-linear mixed } \\
\text { effect modeling using NONMEM }\end{array}$ & Lindbom, L; Pihlgren, P; Jonsson, NE & 2005 & 34.86 \\
\hline 8 & 485 & Lagran program for area and moments in pharmacokinetic analysis & ROCCI, ML; JUSKO, WJ & 1983 & 13.47 \\
\hline 9 & 484 & $\begin{array}{l}\text { PKSolver: An add-in program for pharmacokinetic and pharmacodynamic data analysis } \\
\text { in Microsoft Excel }\end{array}$ & $\begin{array}{l}\text { Zhang, Yong; Huo, Meirong; Zhou, } \\
\text { Jianping; Xie, Shaofei }\end{array}$ & 2010 & 53.78 \\
\hline 10 & 476 & MLAB - mathematical-modeling tool & KNOTT, GD & 1979 & 11.9 \\
\hline 11 & 404 & Kubios HRV - Heart rate variability analysis software & $\begin{array}{l}\text { Tarvainen, Mika P.; Niskanen, Juha- } \\
\text { Pekka; Lipponen, Jukka A.; Ranta-aho, } \\
\text { Perttu O.; Karjalainen, Pasi A. }\end{array}$ & 2014 & 80.8 \\
\hline 12 & 398 & Software for advanced HRV analysis & $\begin{array}{l}\text { Niskanen, JP; Tarvainen, MP; Ranta- } \\
\text { Aho, PO; Karjalainen, PA }\end{array}$ & 2004 & 26.53 \\
\hline 13 & 351 & A review of smart homes - Present state and future challenges & $\begin{array}{l}\text { Chan, Marie; Esteve, Daniel; Escriba, } \\
\text { Christophe; Campo, Eric }\end{array}$ & 2008 & 31.91 \\
\hline 14 & 351 & A monte-carlo program for the simulation of scintillation camera characteristics & LJUNGBERG, M; STRAND, SE & 1989 & 11.7 \\
\hline 15 & 350 & Fast free-form deformation using graphics processing units & $\begin{array}{l}\text { Modat, Marc; Ridgway, Gerard R.; } \\
\text { Taylor, Zeike A.; Lehmann, Manja; } \\
\text { Barnes, Josephine; Hawkes, David J.; } \\
\text { Fox, Nick C.; Ourselin, Sebastien }\end{array}$ & 2010 & 38.89 \\
\hline 16 & 314 & Entropies for detection of epilepsy in EEG & $\begin{array}{l}\text { Kannathal, N; Choo, ML; Acharya, } \\
\text { UR; Sadasivan, PK }\end{array}$ & 2005 & 22.43 \\
\hline 17 & 310 & A computer-program package for relative survival analysis & $\begin{array}{l}\text { HAKULINEN, T; } \\
\text { ABEYWICKRAMA, KH }\end{array}$ & 1985 & 9.12 \\
\hline 18 & 308 & Perls-speaks-NONMEM (PsN) - a Perl module for NONMEM related programming & Lindbom, L; Ribbing, J; Jonsson, NE & 2004 & 20.53 \\
\hline 19 & 297 & Blood vessel segmentation methodologies in retinal images - A survey & $\begin{array}{l}\text { Fraz, M. M.; Remagnino, P.; Hoppe, } \\
\text { A.; Uyyanonvara, B.; Rudnicka, A. R.; } \\
\text { Owen, C. G.; Barman, S. A. }\end{array}$ & 2012 & 42.43 \\
\hline 20 & 294 & MedCalc: A new computer program for medical statistics & $\begin{array}{l}\text { Schoonjans, F; Zalata, A; Depuydt, CE; } \\
\text { Comhaire, FH }\end{array}$ & 1995 & 12.25 \\
\hline 21 & 289 & MIXOR: A computer program for mixed-effects ordinal regression analysis & Hedeker, D; Gibbons, RD & 1996 & 12.57 \\
\hline 22 & 266 & Adjusted survival curves with inverse probability weights & Cole, SR; Hernan, MA & 2004 & 17.73 \\
\hline 23 & 255 & $\begin{array}{l}\text { A procedure for generating bootstrap samples for the validation of nonlinear mixed- } \\
\text { effects population models }\end{array}$ & Parke, J; Holford, NHG; Charles, BG & 1999 & 12.75 \\
\hline 24 & 230 & $\begin{array}{l}\text { CONV - convolution for responses to a finite diameter photon beam incident on multi- } \\
\text { layered tissues }\end{array}$ & Wang, LH; Jacques, SL; Zheng, LQ & 1997 & 10.45 \\
\hline 25 & 229 & $\begin{array}{l}\text { A step-by-step guide to non-linear regression analysis of experimental data using a } \\
\text { Microsoft Excel spreadsheet }\end{array}$ & Brown, AM & 2001 & 12.72 \\
\hline 26 & 229 & $\begin{array}{l}\text { Kruskal-wallis test - basic computer-program to perform nonparametric one-way analysis } \\
\text { of variance and multiple comparisons on ranks of several independent samples }\end{array}$ & THEODORSSONNORHEIM, E & 1986 & 6.94 \\
\hline 27 & 221 & Interactive algorithms for the segmentation and quantitation of 3-D MRI brain scans & Freeborough, PA; Fox, NC; Kitney, RI & 1997 & 10.05 \\
\hline 28 & 214 & Recent development on computer aided tissue engineering - a review & Sun, W; Lal, P & 2002 & 12.59 \\
\hline 29 & 214 & $\begin{array}{l}\text { Structure of the standardized computerized } 24-\mathrm{h} \text { diet recall interview used as reference } \\
\text { method in the } 22 \text { centers participating in the EPIC project }\end{array}$ & $\begin{array}{l}\text { Slimani, N; Deharveng, G; } \\
\text { Charrondiere, RU; van Kappel, AL; } \\
\text { Ocke, MC; Lagiou, A; van Liere, M; } \\
\text { Agudo, A; Pala, V; Brandstetter, B; } \\
\text { Andren, C; Stripp, C; van Staveren, } \\
\text { WA; Riboli, E }\end{array}$ & 1999 & 10.7 \\
\hline 30 & 212 & $\begin{array}{l}\text { Computing normalised prediction distribution errors to evaluate nonlinear mixed-effect } \\
\text { models: The npde add-on package for R }\end{array}$ & $\begin{array}{l}\text { Comets, Emmanuelle; Brendel, Karl; } \\
\text { Mentre, France }\end{array}$ & 2008 & 19.27 \\
\hline 31 & 196 & $\begin{array}{l}\text { MIXREG: A computer program for mixed-effects regression analysis with autocorrelated } \\
\text { errors }\end{array}$ & Hedeker, D; Gibbons, RD & 1996 & 8.52 \\
\hline 32 & 188 & Conversational SAAM - an interactive program for kinetic-analysis of biological-systems & $\begin{array}{l}\text { BOSTON, RC; GREIF, PC; BERMAN, } \\
\text { M }\end{array}$ & 1981 & 4.95 \\
\hline 33 & 180 & MaZda-A software package for image texture analysis & $\begin{array}{l}\text { Szczypinski, Piotr M.; Strzelecki, } \\
\text { Michal; Materka, Andrzej; Klepaczko, } \\
\text { Artur }\end{array}$ & 2009 & 18 \\
\hline 34 & 180 & Classification of EEG signals using neural network and logistic regression & Subasi, A; Ercelebi, E & 2005 & 12.86 \\
\hline 35 & 165 & $\begin{array}{l}\text { Gaining more flexibility in Cox proportional hazards regression models with cubic spline } \\
\text { functions }\end{array}$ & Heinzl, H; Kaider, A & 1997 & 7.5 \\
\hline
\end{tabular}




\begin{tabular}{|c|c|c|c|c|c|}
\hline 36 & 157 & Computerized collection and analysis of dietary-intake information & $\begin{array}{l}\text { FESKANICH, D; SIELAFF, BH; } \\
\text { CHONG, K; BUZZARD, IM }\end{array}$ & 1989 & 5.23 \\
\hline 37 & 149 & Non-linear analysis of EEG signals at various sleep stages & $\begin{array}{l}\text { Acharya, R; Faust, O; Kannathal, N; } \\
\text { Chua, T; Laxminarayan, S }\end{array}$ & 2005 & 10.64 \\
\hline 38 & 142 & Skin photoplethysmography - a review & $\begin{array}{l}\text { KAMAL, AAR; HARNESS, JB; } \\
\text { IRVING, G; MEARNS, AJ }\end{array}$ & 1989 & 4.73 \\
\hline 39 & 142 & $\begin{array}{l}\text { Computer-programs for the radioactive microsphere technique - determination of regional } \\
\text { blood flows and other hemodynamic variables in different experimental circumstances }\end{array}$ & $\begin{array}{l}\text { SAXENA, PR; SCHAMHARDT, HC; } \\
\text { FORSYTH, RP; HOEVE, J }\end{array}$ & 1980 & 3.64 \\
\hline 40 & 142 & A program for non-linear regression-analysis to be used on desk-top computers & KOEPPE, P; HAMANN, C & 1980 & 3.64 \\
\hline 41 & 139 & Real-time deformable models for surgery simulation: a survey & $\begin{array}{l}\text { Meier, U; Lopez, O; Monserrat, C; } \\
\text { Juan, MC; Alcaniz, M }\end{array}$ & 2005 & 9.93 \\
\hline 42 & 138 & $\begin{array}{l}\text { DAISY: A new software tool to test global identifiability of biological and physiological } \\
\text { systems }\end{array}$ & $\begin{array}{l}\text { Bellu, Giuseppina; Saccomani, Maria } \\
\text { Pia; Audoly, Stefania; D'Angio, } \\
\text { Leontina }\end{array}$ & 2007 & 11.5 \\
\hline 43 & 137 & $\begin{array}{l}\text { A SAS macro for estimation of direct adjusted survival curves based on a stratified Cox } \\
\text { regression model }\end{array}$ & $\begin{array}{l}\text { Zhang, Xu; Loberiza, Fausto R.; Klein, } \\
\text { John P.; Zhang, Mei-Jie }\end{array}$ & 2007 & 11.42 \\
\hline 44 & 133 & Pirana and PCluster: A modeling environment and cluster infrastructure for NONMEM & $\begin{array}{l}\text { Keizer, Ron J.; van Benten, Michel; } \\
\text { Beijnen, Jos H.; Schellens, Jan H. M.; } \\
\text { Huitema, Alwin D. R. }\end{array}$ & 2011 & 16.63 \\
\hline 45 & 132 & MASTER: a Windows program for recording multiple auditory steady-state responses & John, MS; Picton, TW & 2000 & 6.95 \\
\hline 46 & 131 & A review of atlas-based segmentation for magnetic resonance brain images & $\begin{array}{l}\text { Cabezas, Mariano; Oliver, Arnau; } \\
\text { Llado, Xavier; Freixenet, Jordi; Cuadra, } \\
\text { Meritxell Bach }\end{array}$ & 2011 & 16.38 \\
\hline 47 & 123 & $\begin{array}{l}\text { MIC-II - program for determination of cardiac-output, arteriovenous shunt and regional } \\
\text { blood-flow using radioactive microsphere method }\end{array}$ & $\begin{array}{l}\text { SCHOSSER, R; ARFORS, KE; } \\
\text { MESSMER, K }\end{array}$ & 1979 & 3.08 \\
\hline 48 & 119 & ECG beat classification by a novel hybrid neural network & Dokur, Z; Olmez, T & 2001 & 6.61 \\
\hline 49 & 116 & $\begin{array}{l}\text { A state of the art review on intima-media thickness (IMT) measurement and wall } \\
\text { segmentation techniques for carotid ultrasound }\end{array}$ & $\begin{array}{l}\text { Molinari, Filippo; Zeng, Guang; Suri, } \\
\text { Jasjit S. }\end{array}$ & 2010 & 12.89 \\
\hline 50 & 115 & Characterization of EEG - A comparative study & $\begin{array}{l}\text { Kannathal, N; Acharya, UR; Lim, CM; } \\
\text { Sadasivan, P }\end{array}$ & 2005 & 8.21 \\
\hline
\end{tabular}


Table 3. Top 40 most cited documents in CMPB publications

\begin{tabular}{|c|c|c|c|c|c|c|c|c|}
\hline Rank & Year & First author & Reference & Vol & Page & Type & $\mathrm{TC}$ & $\begin{array}{c}\text { Co- } \\
\text { citations }\end{array}$ \\
\hline 1 & 2000 & Goldberger AL & Circulation & v101 & pe215 & A & 50 & 32 \\
\hline 2 & 1972 & Cox DR & J R Stat Soc B & v34 & p187 & A & 48 & 19 \\
\hline 3 & 1973 & Haralick RM & IEEE T Syst Man Cyb & v3 & p610 & A & 45 & 34 \\
\hline 4 & 1979 & Otsu N & IEEE T Syst Man Cyb & v9 & p62 & A & 45 & 29 \\
\hline 5 & 2001 & Breiman L & Mach Learn & $\mathrm{v} 45$ & p5 & A & 33 & 30 \\
\hline 6 & 1995 & Cortes C & Mach Learn & v20 & p273 & A & 31 & 26 \\
\hline 7 & 1974 & Akaike $\mathrm{H}$ & IEEE T Automat Contr & v19 & p716 & A & 30 & 17 \\
\hline 8 & 1985 & Pan J & IEEE T Bio-Med Eng & v32 & p230 & A & 30 & 21 \\
\hline 9 & 1963 & Marquardt DW & J Soc Ind Appl Math & v11 & p431 & A & 27 & 16 \\
\hline 10 & 1991 & Pincus SM & P Natl Acad Sci Usa & v88 & p2297 & A & 26 & 20 \\
\hline 11 & 2001 & Chan TF & IEEE T Image Process & v10 & p266 & A & 25 & 20 \\
\hline 12 & 2001 & Duda RO & Pattern Classificati & & & B & 25 & 17 \\
\hline 13 & 1987 & Lorensen WE & Comput Graph & v21 & p163 & A & 25 & 10 \\
\hline 14 & 2005 & Witten IH & Data Mining Practica & & & B & 24 & 14 \\
\hline 15 & 2011 & Chang CC & ACM T Intel Syst Tec & v2 & & A & 23 & 19 \\
\hline 16 & 1977 & Dempster AP & J Roy Stat Soc B Met & v39 & $\mathrm{p} 1$ & A & 23 & 14 \\
\hline 17 & 1989 & Mallat SG & IEEE T Pattern Anal & v11 & p674 & A & 23 & 13 \\
\hline 18 & 1998 & Burges CJC & Data Min Knowl Disc & v2 & p121 & A & 22 & 19 \\
\hline 19 & 1983 & Carson ER & Math Modeling Metabo & & & B & 22 & 13 \\
\hline 20 & 2004 & Hovorka R & Physiol Meas & v25 & p905 & A & 21 & 14 \\
\hline 21 & 1996 & Breiman L & Mach Learn & v24 & p123 & A & 20 & 20 \\
\hline 22 & 1973 & Duda RO & Pattern Classificati & & & B & 20 & 11 \\
\hline 23 & 2008 & Gonzalez RC & Digital Image Proces & & & B & 20 & 14 \\
\hline 24 & 1987 & Kass M & Int J Comput Vision & v1 & p321 & A & 20 & 17 \\
\hline 25 & 1965 & Nelder JA & Comput J & v7 & p308 & A & 20 & 8 \\
\hline 26 & 1996 & Camm AJ & Circulation & v93 & p1043 & A & 19 & 16 \\
\hline 27 & 2005 & Hann CE & Comput Meth Prog Bio & v77 & p259 & A & 19 & 16 \\
\hline 28 & 1998 & Huang NE & P Roy Soc A-Math Phy & v454 & p903 & A & 19 & 14 \\
\hline 29 & 2000 & Richman JS & Am J Physiol-Heart C & v278 & ph2039 & A & 19 & 19 \\
\hline 30 & 2004 & Staal J & IEEE T Med Imaging & v23 & p501 & A & 19 & 15 \\
\hline 31 & 1998 & Vapnik V & Stat Learning Theory & & & B & 19 & 14 \\
\hline 32 & 1986 & Bland JM & Lancet & v1 & p307 & A & 18 & 7 \\
\hline 33 & 1984 & Breiman L & Classification Regre & & & B & 18 & 14 \\
\hline 34 & 1982 & Hanley JA & Radiology & v143 & p29 & A & 18 & 10 \\
\hline 35 & 1986 & Liang KY & Biometrika & v73 & p13 & A & 18 & 6 \\
\hline 36 & 1998 & Maintz JB & Med Image Anal & v2 & p1 & A & 18 & 15 \\
\hline 37 & 1992 & Press WH & Numerical Recipes C & & & B & 18 & 7 \\
\hline 38 & 1999 & Rueckert D & IEEE T Med Imaging & v18 & p712 & A & 18 & 15 \\
\hline 39 & 1986 & Canny J & IEEE T Pattern Anal & v8 & p679 & A & 17 & 14 \\
\hline 40 & 1989 & Chaudhuri S & IEEE T Med Imaging & $\mathrm{v} 8$ & p263 & A & 17 & 16 \\
\hline
\end{tabular}


Table 4. Top 50 leading authors in CMPB

\begin{tabular}{|c|c|c|c|c|c|c|c|c|c|c|}
\hline $\mathrm{R}$ & Author Name & University & Country & $\mathrm{TP}$ & TC & $\mathrm{H}$ & $\mathrm{C} / \mathrm{P}$ & $\geq 200$ & $\geq 100$ & $\geq 50$ \\
\hline 1 & James Geoffrey Chase & U Canterbury & New Zealand & 40 & 650 & 15 & 16.25 & 1 & 3 & 18 \\
\hline 2 & & Christchurch Hospital, & & & & & & & & \\
\hline 2 & Geoffrey M. Shaw & Christchurch & New Zealand & 32 & 545 & 14 & 17.03 & 1 & 2 & 15 \\
\hline 3 & Yu-Chuan Li & Taipei Medical U & Taiwan & 28 & 222 & 9 & 7.93 & 0 & 0 & 6 \\
\hline 4 & Christopher E. Hann & U Canterbury & New Zealand & 24 & 536 & 14 & 22.33 & 1 & 3 & 14 \\
\hline 5 & Arie Hasman & Reactor Institute Delft & Netherlands & 23 & 193 & 8 & 8.39 & 0 & 0 & 5 \\
\hline 6 & Steen Andreassen & Aalborg U & Denmark & 21 & 303 & 10 & 14.43 & 0 & 1 & 10 \\
\hline 7 & Marco Viceconti & Insigneo: Inst for in silico medicine & UK & 20 & 308 & 10 & 15.40 & 0 & 0 & 10 \\
\hline 8 & Torgny L. Groth & Uppsala U & Sweden & 19 & 192 & 8 & 10.11 & 0 & 0 & 6 \\
\hline 9 & Jian Wang & BioFortis, Inc. & USA & 18 & 169 & 9 & 9.39 & 0 & 0 & 6 \\
\hline 10 & Usman Iqbal & Taipei Medical U & Taiwan & 16 & 102 & 6 & 6.38 & 0 & 0 & 4 \\
\hline 11 & Jasjit S. Suri & Global Biomedical Technologies & USA & 15 & 404 & 10 & 26.93 & 1 & 2 & 10 \\
\hline 12 & Roman Hovorka & U Cambridge & UK & 15 & 305 & 8 & 20.33 & 0 & 2 & 7 \\
\hline 13 & Debora Testi & CINECA & Italy & 15 & 226 & 9 & 15.07 & 0 & 0 & 7 \\
\hline 14 & Hans Peter Meinzer & German Cancer Research Center & Germany & 13 & 129 & 6 & 9.92 & 0 & 1 & 3 \\
\hline 15 & James F. Reed III & Christiana Care Health System & USA & 13 & 82 & 5 & 6.31 & 0 & 0 & 2 \\
\hline 16 & Derek Arthur Linkens & U Sheffield & UK & 13 & 76 & 5 & 5.85 & 0 & 0 & 1 \\
\hline 17 & Michael J. Chappell & U Warwick & UK & 13 & 72 & 4 & 5.54 & 0 & 0 & 2 \\
\hline 18 & Jessica Lin & U Otago & New Zealand & 12 & 324 & 7 & 27.00 & 1 & 2 & 6 \\
\hline 19 & Ewart R. Carson & City U London & UK & 12 & 258 & 10 & 21.50 & 0 & 1 & 10 \\
\hline 20 & Phung Anh (Alex) Nguyen & Taipei Medical U & Taiwan & 12 & 75 & 5 & 6.25 & 0 & 0 & 3 \\
\hline 21 & Mohammad A.A. Moussa & Kuwait U & Kuwait & 12 & 32 & 3 & 2.67 & 0 & 0 & 0 \\
\hline 22 & U Rajendra Acharya & Ngee Ann Polytechnic & Singapore & 11 & 656 & 10 & 59.64 & 2 & 3 & 10 \\
\hline 23 & Claudio Cobelli & U Padua & Italy & 11 & 293 & 9 & 26.64 & 0 & 2 & 9 \\
\hline 24 & Thomas Desaive & U Liège & Belgium & 11 & 120 & 5 & 10.91 & 0 & 0 & 4 \\
\hline 25 & Ove B. Wigertz & Linköping U & Sweden & 11 & 89 & 7 & 8.09 & 0 & 0 & 2 \\
\hline 26 & Kiyonari Inamura & Osaka U & Japan & 11 & 49 & 4 & 4.45 & 0 & 0 & 1 \\
\hline 27 & Martti Juhola & U Tampere & Finland & 10 & 99 & 7 & 9.90 & 0 & 0 & 3 \\
\hline 28 & Cinzia Zannoni & CINECA & Italy & 9 & 185 & 7 & 20.56 & 0 & 0 & 6 \\
\hline 29 & Tibor Deutsch & Applied Logic Laboratory & Hungary & 9 & 179 & 7 & 19.89 & 0 & 0 & 7 \\
\hline 30 & Ewert Bengtsson & Uppsala U & Sweden & 9 & 102 & 5 & 11.33 & 0 & 1 & 1 \\
\hline 31 & Uwe Engelmann & CHILI GmbH & Germany & 9 & 74 & 5 & 8.22 & 0 & 0 & 2 \\
\hline 32 & Syed-Abdul Shabbir & Taipei Medical U & Taiwan & 9 & 56 & 4 & 6.22 & 0 & 0 & 1 \\
\hline 33 & Kouhei Akazawa & National U Niigata & Japan & 9 & 35 & 4 & 3.89 & 0 & 0 & 1 \\
\hline 34 & Angus M. Brown & U Nottingham & UK & 8 & 316 & 6 & 39.50 & 1 & 1 & 5 \\
\hline 35 & Maurizio Rocchetti & Independent Consultant & Italy & 8 & 209 & 6 & 26.13 & 0 & 2 & 3 \\
\hline 36 & Riccardo Bellazzi & U Pavia & Italy & 8 & 195 & 7 & 24.38 & 0 & 2 & 4 \\
\hline 37 & Roberto Hornero & U Valladolid & Spain & 8 & 191 & 5 & 23.88 & 0 & 1 & 4 \\
\hline 38 & Atam Prakash Dhawan & New Jersey Institute of Technology & USA & 8 & 188 & 8 & 23.50 & 0 & 0 & 8 \\
\hline 39 & Mario Stefanelli & U Pavia & Italy & 8 & 188 & 7 & 23.50 & 0 & 2 & 3 \\
\hline 40 & Ole Kristian Hejlesen & Aalborg U & Denmark & 8 & 165 & 7 & 20.63 & 0 & 1 & 4 \\
\hline 41 & Peter Sonksen & U Southampton & UK & 8 & 157 & 8 & 19.63 & 0 & 0 & 7 \\
\hline 42 & Yongtian Wang & Beijing Institute of Technology & PR China & 8 & 102 & 5 & 12.75 & 0 & 0 & 2 \\
\hline 43 & Georgios C. Nikiforidis & U Patras & Greece & 8 & 89 & 4 & 11.13 & 0 & 0 & 3 \\
\hline 44 & Ming Huei Hsu & Taipei Medical U & Taiwan & 8 & 82 & 5 & 10.25 & 0 & 0 & 2 \\
\hline 45 & Herman P. Wijnand & Herman P. Wijnand & Israel & 8 & 80 & 6 & 10.00 & 0 & 0 & 3 \\
\hline 46 & Jeongjin Lee & Soongsil U & South Korea & 8 & 70 & 2 & 8.75 & 0 & 1 & 1 \\
\hline 47 & Nobutaka Ikeda & Toho U & Japan & 8 & 64 & 5 & 8.00 & 0 & 0 & 2 \\
\hline 48 & Sanghoon Lee & Yonsei U & South Korea & 8 & 61 & 3 & 7.63 & 0 & 0 & 2 \\
\hline 49 & Patrice Degoulet & Hop Europe Georges-Pompidou & France & 8 & 41 & 4 & 5.13 & 0 & 0 & 1 \\
\hline 50 & Neil Evans & U Warwick & UK & 8 & 36 & 4 & 4.50 & 0 & 0 & 0 \\
\hline
\end{tabular}


Table 5. Temporal evolution of the most productive authors

\begin{tabular}{|c|c|c|c|c|c|c|c|}
\hline $\mathrm{R}$ & Author & $\mathrm{TP}$ & TC & $\mathrm{R}$ & Author & $\mathrm{TP}$ & TC \\
\hline \multicolumn{4}{|c|}{ 1970-1977 } & \multicolumn{4}{|c|}{$1998-2007$} \\
\hline 1 & Elisa T Lee & 25 & 877 & 1 & Viceconti M & 16 & 293 \\
\hline 2 & John R. Cunningham & 7 & 105 & 2 & Chase JG & 11 & 290 \\
\hline 3 & Jan Van De Geijn & 6 & 99 & 3 & Testi D & 11 & 213 \\
\hline 4 & Andrew S. French & 6 & 77 & 4 & Shaw GM & 10 & 237 \\
\hline 5 & Jan E. Ekstedt & 4 & 59 & 5 & Zannoni C & 9 & 185 \\
\hline 6 & Horowitz JM & 4 & 7 & 6 & Hann CE & 8 & 247 \\
\hline \multicolumn{4}{|c|}{ 1978-1987 } & 7 & Cappello A & 7 & 147 \\
\hline 1 & Moussa MAA & 10 & 26 & 8 & Akazawa K & 7 & 26 \\
\hline 2 & Groves WE & 7 & 12 & 9 & Brown AM & 6 & 305 \\
\hline 3 & Begole EA & 6 & 26 & 10 & Gomez EJ & 6 & 228 \\
\hline 4 & Rocchetti M & 5 & 184 & 11 & Andreassen S & 6 & 94 \\
\hline 5 & Okada M & 5 & 7 & 12 & $\mathrm{Xu}$ YH & 6 & 75 \\
\hline 6 & Recchia M & 4 & 108 & 13 & Kannathal N & 5 & 616 \\
\hline 7 & Gath I & 4 & 60 & 14 & Bellazzi R & 5 & 173 \\
\hline 8 & Miller PL & 4 & 55 & 15 & Fischer G & 5 & 110 \\
\hline 9 & Larsen SO & 4 & 28 & 16 & Tilg B & 5 & 110 \\
\hline 10 & Tyson $\mathrm{H}$ & 4 & 14 & 17 & Baruffaldi $F$ & 5 & 68 \\
\hline 11 & Horowitz JM & 4 & 10 & 18 & Pitot $\mathrm{HC}$ & 5 & 51 \\
\hline 12 & Duisterhout JS & 4 & 8 & 19 & Hasman A & 5 & 38 \\
\hline \multicolumn{4}{|c|}{$1988-1997$} & 20 & Inoue $\mathrm{M}$ & 5 & 26 \\
\hline 1 & Hasman A & 17 & 133 & \multicolumn{4}{|c|}{$2008-2017$} \\
\hline 2 & Groth T & 12 & 103 & 1 & Chase JG & 29 & 360 \\
\hline 3 & Hovorka R & 10 & 259 & 2 & $\mathrm{Li} \mathrm{YC}$ & 27 & 189 \\
\hline 4 & Wigertz O & 10 & 79 & 3 & Shaw GM & 22 & 308 \\
\hline 5 & Reed JF & 10 & 56 & 4 & Hann CE & 16 & 289 \\
\hline 6 & Carson ER & 8 & 208 & 5 & Iqbal U & 16 & 102 \\
\hline 7 & Engelmann U & 8 & 52 & 6 & Suri JS & 15 & 411 \\
\hline 8 & Degoulet P & 8 & 41 & 7 & Jian WS & 15 & 96 \\
\hline 9 & Inamura $\mathrm{K}$ & 8 & 27 & 8 & Nguyen PA & 12 & 75 \\
\hline 10 & Sonksen PH & 7 & 144 & 9 & Chappell MJ & 12 & 58 \\
\hline 11 & Andreassen $\mathrm{S}$ & 7 & 142 & 10 & Lin J & 10 & 195 \\
\hline 12 & Meinzer HP & 7 & 44 & 11 & Desaive $\mathrm{T}$ & 10 & 96 \\
\hline 13 & Jean FC & 7 & 42 & 12 & Syed-Abdul S & 9 & 56 \\
\hline 14 & Brender J & 6 & 66 & 13 & Acharya UR & 8 & 221 \\
\hline 15 & Schroter A & 6 & 43 & 14 & Andreassen S & 8 & 67 \\
\hline 16 & Grimson W & 6 & 31 & 15 & Evans ND & 8 & 36 \\
\hline 17 & Theodoropoulos G & 6 & 25 & 16 & Hornero R & 7 & 163 \\
\hline 18 & Kormano M & 6 & 17 & 17 & Saba L & 7 & 108 \\
\hline
\end{tabular}

Abbreviations are available in the previous tables.

Another set of analyses (shown in Table 6) provides information about the most productive and influential institutions (in terms of cites/paper and university rankings) in CMPB. The top 3 institutions in the list are Uppsala U (Sweden), Taipei Medical U(Taiwan) and INSERM (France). The highest number of institutions in the list is from the USA with top ranked institutes like Harvard University, Stanford University, UCLA and Yale University. North American and European institutions dominate in this list and only a few institutions are from 
Asia (South Korea, Taiwan and China). If we look at the temporal evolution of institutions since the early days of the journal, US and European institutions dominated the list (19781987) with an exception of Kuwait University. In the last 10 years, there is a number of institutions from Asia (Taipei Medical U, National Yang Ming U, Shanghai Jiao Tong U, Seoul National U, National Taiwan U, Chinese Academy of Sci, National Taiwan U Sci Tech, National U Singapore, Amirkabir U Technology, National U Sci Tech Pakistan, Nanyang Technological U, China Medical U Taiwan), South America (U Est Rio de Janeiro) and Oceania (U Canterbury) have started to come up in the list as well which is overall still very much dominated by European and US institutions.

To get an overall summary of the contributions to CMPB, we have analysed the publications at the country level (see Table 8). In terms of the most number of papers, the USA, the UK and Italy occupy the top 3 positions with only PR China and Taiwan appearing in the top 10 list from outside North America and Europe. However, if we analyse these results on a per capita basis, Sweden, Finland, Slovenia, Denmark and New Zealand are the top most productive countries. Further, if we look at the citations per capita, Sweden and Finland top the list with Singapore, New Zealand and Slovenia occupying the next three positions. Overall, it is evident that CMPB publications are diverse and are coming from all over the world. This shows the reach and overall standing of this journal in different parts of the world. If we look at the trend of papers coming from each of these countries annually (see Table 9), we find that papers from countries like China, Taiwan, Spain, Italy, UK are rapidly increasing while contributions from USA have been stable over the last few decades. If we just look at the papers from the last decade (2017-2008), we observe that the number of papers from PR China is increasing and it has overtaken the US in recent years (2017 and 2016). 
Table 6. The most productive and influential institutions in CMPB

\begin{tabular}{|c|c|c|c|c|c|c|c|c|c|c|c|}
\hline $\mathrm{R}$ & Institution & Country & TP & $\mathrm{TC}$ & $\mathrm{H}$ & $\mathrm{C} / \mathrm{P}$ & $\geq 250$ & $\geq 100$ & $\geq 50$ & $\mathrm{QS}$ & ARWU \\
\hline 1 & Uppsala U & Sweden & 77 & 2232 & 15 & 28.99 & 3 & 6 & 28 & 112 & 63 \\
\hline 2 & Taipei Medical U & Taiwan & 61 & 411 & 12 & 6.74 & 0 & 0 & 13 & 362 & - \\
\hline 3 & INSERM & France & 58 & 1082 & 17 & 18.66 & 2 & 3 & 27 & - & - \\
\hline 4 & CNR & Italy & 56 & 953 & 11 & 17.02 & 1 & 1 & 12 & - & - \\
\hline 5 & Harvard U & USA & 47 & 820 & 15 & 17.45 & 1 & 2 & 18 & 38 & 1 \\
\hline 6 & U California Davis & USA & 45 & 255 & 8 & 5.67 & 0 & 1 & 6 & 3 & 96 \\
\hline 7 & CNRS & France & 42 & 1083 & 18 & 25.79 & 1 & 3 & 25 & 100 & - \\
\hline 8 & Assistance Publ Hop Paris & France & 42 & 856 & 14 & 20.38 & 2 & 3 & 17 & - & - \\
\hline 9 & U Canterbury & New Zealand & 42 & 662 & 15 & 15.76 & 1 & 3 & 19 & 231 & $401-500$ \\
\hline 10 & National Inst Health & USA & 39 & 1155 & 12 & 29.62 & 2 & 4 & 14 & - & - \\
\hline 11 & National Yang Ming U & Taiwan & 39 & 253 & 9 & 6.49 & 0 & 0 & 8 & 292 & $501-600$ \\
\hline 12 & Helmholtz Association & Germany & 37 & 389 & 10 & 10.51 & 0 & 1 & 10 & - & - \\
\hline 13 & U Politec Valencia & Spain & 35 & 544 & 13 & 15.54 & 1 & 2 & 16 & $561-570$ & $401-500$ \\
\hline 14 & Erasmus U Rotterdam & Netherlands & 34 & 440 & 9 & 12.94 & 1 & 3 & 9 & 147 & 73 \\
\hline 15 & U Washington Seattle & USA & 33 & 598 & 12 & 18.12 & 1 & 2 & 15 & 66 & 14 \\
\hline 16 & Maastricht U & Netherlands & 32 & 244 & 9 & 7.63 & 0 & 0 & 7 & 211 & $201-300$ \\
\hline 17 & U Padua & Italy & 30 & 607 & 12 & 20.23 & 1 & 4 & 13 & 296 & $151-200$ \\
\hline 18 & Linkoping U & Sweden & 30 & 315 & 10 & 10.5 & 0 & 1 & 9 & 302 & $301-400$ \\
\hline 19 & City U London & UK & 29 & 526 & 14 & 18.14 & 0 & 2 & 15 & 343 & - \\
\hline 20 & Aalborg U & Denmark & 29 & 434 & 13 & 14.97 & 0 & 2 & 13 & 343 & $201-300$ \\
\hline 21 & U Toronto & Canada & 29 & 420 & 8 & 14.48 & 1 & 2 & 5 & 31 & 23 \\
\hline 22 & Christchurch Hospital & New Zealand & 28 & 470 & 11 & 16.79 & 1 & 2 & 12 & - & - \\
\hline 23 & German Cancer Res Center & Germany & 27 & 273 & 8 & 10.11 & 0 & 1 & 7 & - & - \\
\hline 24 & U Pittsburgh & USA & 27 & 202 & 8 & 7.48 & 0 & 0 & 7 & 136 & 68 \\
\hline 25 & Karolinska Instit & Sweden & 26 & 476 & 10 & 18.31 & 1 & 2 & 8 & - & - \\
\hline 26 & Taipei Municipal Wanfang Hosp & Taiwan & 26 & 140 & 7 & 5.38 & 0 & 0 & 2 & - & - \\
\hline 27 & Irces Istituto Ortopedico Rizzoli & Italy & 25 & 364 & 11 & 14.56 & 0 & 0 & 11 & - & - \\
\hline 28 & Seoul National U & South Korea & 25 & 261 & 9 & 10.44 & 0 & 1 & 8 & 36 & $101-150$ \\
\hline 29 & National Taiwan U & Taiwan & 25 & 195 & 8 & 7.8 & 0 & 0 & 7 & 72 & $151-200$ \\
\hline 30 & U Bologna & Italy & 24 & 305 & 10 & 12.71 & 0 & 0 & 10 & 188 & $201-300$ \\
\hline 31 & Stanford U & USA & 24 & 211 & 7 & 8.79 & 0 & 1 & 5 & 2 & 2 \\
\hline 32 & U Patras & Greece & 24 & 191 & 7 & 7.96 & 0 & 0 & 7 & $701-750$ & $901-1000$ \\
\hline 33 & U Turku & Finland & 24 & 180 & 7 & 7.5 & 0 & 0 & 6 & 285 & $301-400$ \\
\hline 34 & Johns Hopkins U & USA & 23 & 1157 & 10 & 50.3 & 2 & 2 & 10 & 21 & 18 \\
\hline 35 & U Sheffield & UK & 23 & 182 & 7 & 7.91 & 0 & 1 & 3 & 75 & $101-150$ \\
\hline 36 & U Munich & Germany & 22 & 274 & 7 & 12.45 & 1 & 1 & 6 & 61 & 53 \\
\hline 37 & VTT Technical Research Center & Finland & 22 & 196 & 7 & 8.91 & 0 & 1 & 4 & 156 & $201-300$ \\
\hline 38 & U California Los Angeles & USA & 22 & 85 & 5 & 3.86 & 0 & 0 & 1 & 32 & 11 \\
\hline 39 & Yale U & USA & 21 & 951 & 9 & 45.29 & 2 & 2 & 7 & 15 & 12 \\
\hline 40 & U Pavia & Italy & 21 & 278 & 9 & 13.24 & 0 & 2 & 7 & $581-590$ & $301-400$ \\
\hline 41 & U Michigan & USA & 21 & 274 & 7 & 13.05 & 1 & 1 & 7 & 20 & 24 \\
\hline 42 & Ruprecht Karls U Heidelberg & Germany & 21 & 272 & 9 & 12.95 & 0 & 1 & 6 & 64 & 47 \\
\hline 43 & U Tampere & Finland & 21 & 246 & 10 & 11.71 & 0 & 0 & 10 & 366 & $601-700$ \\
\hline 44 & Polytechnic U Milan & Italy & 21 & 146 & 7 & 6.95 & 0 & 0 & 5 & 170 & $201-300$ \\
\hline 45 & U Warwick & UK & 21 & 142 & 7 & 6.76 & 0 & 0 & 3 & 57 & $101-150$ \\
\hline 46 & National U Singapore & Singapore & 20 & 591 & 9 & 29.55 & 2 & 2 & 9 & 15 & 91 \\
\hline 47 & U Ljubljana & Slovenia & 20 & 263 & 8 & 13.15 & 0 & 1 & 7 & $651-700$ & $401-500$ \\
\hline 48 & Sapienza U Rome & Italy & 20 & 152 & 8 & 7.6 & 0 & 0 & 6 & 215 & $151-200$ \\
\hline 49 & Shanghai Jiao Tong U & China & 19 & 359 & 9 & 18.89 & 0 & 2 & 8 & 59 & $101-150$ \\
\hline 50 & Polytechnic U Madrid & Spain & 19 & 353 & 10 & 18.58 & 0 & 2 & 10 & 470 & $501-600$ \\
\hline
\end{tabular}


Table 7. Temporal evolution of the most productive institutions

\begin{tabular}{|c|c|c|c|c|c|c|c|}
\hline $\mathrm{R}$ & Institution & $\mathrm{TP}$ & $\mathrm{TC}$ & $\mathrm{R}$ & Institution & $\mathrm{TP}$ & TC \\
\hline \multicolumn{4}{|c|}{ 1978-1987 } & \multicolumn{4}{|c|}{$2008-2017$} \\
\hline 1 & U California Davis & 22 & 122 & 1 & Taipei Medical U & 58 & 340 \\
\hline 2 & Erasmus U Rotterdam & 19 & 258 & 2 & National Yang Ming U & 35 & 186 \\
\hline 3 & Vrije U Amsterdam & 15 & 27 & 3 & CNRS & 29 & 931 \\
\hline 4 & INSERM & 12 & 80 & 4 & U Canterbury & 29 & 360 \\
\hline 5 & Kuwait U & 12 & 30 & 5 & U Politec Valencia & 28 & 280 \\
\hline 6 & U Michigan & 11 & 101 & 6 & National Taiwan U & 23 & 172 \\
\hline 7 & German Cancer Res Center & 10 & 71 & 7 & INSERM & 22 & 531 \\
\hline 8 & Helmholtz Association & 10 & 71 & 8 & Istit Naz di Fisica Nucleare & 21 & 138 \\
\hline 9 & Medical U South Carolina & 10 & 25 & 9 & U Warwick & 20 & 128 \\
\hline \multicolumn{4}{|c|}{$1988-1997$} & 10 & CNR Italy & 18 & 86 \\
\hline 1 & Uppsala U & 31 & 365 & 11 & Seoul National U & 17 & 111 \\
\hline 2 & CNR Italy & 27 & 193 & 12 & Harvard U & 16 & 133 \\
\hline 3 & Maastricht U & 22 & 180 & 13 & U Porto & 15 & 173 \\
\hline 4 & Linkoping U & 19 & 192 & 14 & U Girona & 14 & 203 \\
\hline 5 & Istit Naz di Fisica Nucleare & 19 & 103 & 15 & Shanghai Jiao Tong U & 14 & 177 \\
\hline 6 & Hasselt U & 17 & 144 & 16 & U Patras & 14 & 117 \\
\hline 7 & U Turku & 17 & 115 & 17 & U Padua & 14 & 113 \\
\hline 8 & Vtt Tech Res Center Finland & 16 & 151 & 18 & Idaho State U & 13 & 377 \\
\hline 9 & City U London & 14 & 366 & 19 & U Valladolid & 13 & 285 \\
\hline 10 & INSERM & 14 & 160 & 20 & Uppsala U & 13 & 236 \\
\hline 11 & Karolinska Instit & 11 & 121 & 21 & U Otago & 12 & 236 \\
\hline 12 & German Cancer Res Center & 11 & 105 & 22 & U Athens & 12 & 116 \\
\hline 13 & Osaka U & 11 & 37 & 23 & U da Coruna & 12 & 112 \\
\hline 14 & Guy S St Thomas NHS Found Trust & 10 & 222 & 24 & Aalborg U & 12 & 95 \\
\hline 15 & Aalborg U & 10 & 159 & 25 & Chinese Academy of Sci & 12 & 81 \\
\hline 16 & Erasmus U Rotterdam & 10 & 148 & 26 & National Taiwan U Sci Tech & 12 & 37 \\
\hline \multicolumn{4}{|c|}{$1998-2007$} & 27 & U Est Rio de Janeiro & 11 & 120 \\
\hline 1 & Istit Naz di Fisica Nucleare & 21 & 458 & 28 & Ghent U & 11 & 110 \\
\hline 2 & Irces Istit Ortopedico Rizzoli & 19 & 317 & 29 & National U Singapore & 11 & 106 \\
\hline 3 & U Washington Seattle & 15 & 438 & 30 & Polytechnic U Madrid & 11 & 89 \\
\hline 4 & Uppsala U & 13 & 1552 & 31 & Ciber Centro de Inv Biomed & 11 & 70 \\
\hline 5 & Harvard U & 12 & 509 & 32 & Amirkabir U Technology & 10 & 230 \\
\hline 6 & U Ljubljana & 11 & 810 & 33 & National U Sci Tech Pakistan & 10 & 124 \\
\hline 7 & U Padua & 11 & 427 & 34 & Nanyang Technological U & 10 & 119 \\
\hline 8 & U Canterbury & 11 & 290 & 35 & U Pittsburgh & 10 & 104 \\
\hline 9 & U Bologna & 11 & 225 & 36 & U Liege & 10 & 96 \\
\hline 10 & City U London & 11 & 145 & 37 & Sapienza U Rome & 10 & 85 \\
\hline \multirow[t]{3}{*}{11} & U Munich & 10 & 82 & 38 & U Vigo & 10 & 85 \\
\hline & & & & 39 & U Sao Paulo & 10 & 68 \\
\hline & & & & 40 & China Medical U Taiwan & 10 & 58 \\
\hline
\end{tabular}

Abbreviations are available in the previous tables. 
Table 8. The most productive and influential countries in CMPB

\begin{tabular}{|c|c|c|c|c|c|c|c|c|}
\hline $\mathrm{R}$ & Country & $\mathrm{TP}$ & $\mathrm{TC}$ & $\mathrm{H}$ & $\mathrm{C} / \mathrm{P}$ & Population & $\mathrm{P} / \mathrm{Po}$ & $\mathrm{C} / \mathrm{Po}$ \\
\hline 1 & USA & 1098 & 17578 & 45 & 16.01 & 324118787 & 3.39 & 54.23 \\
\hline 2 & United Kingdom & 333 & 5081 & 33 & 15.26 & 65640000 & 5.07 & 77.41 \\
\hline 3 & Italy & 305 & 3891 & 28 & 12.76 & 59801004 & 5.1 & 65.07 \\
\hline 4 & Germany & 296 & 3405 & 29 & 11.5 & 80682351 & 3.67 & 42.2 \\
\hline 5 & Spain & 226 & 2773 & 27 & 12.27 & 46064604 & 4.91 & 60.2 \\
\hline 6 & France & 225 & 3882 & 30 & 17.25 & 64668129 & 3.48 & 60.03 \\
\hline 7 & Peoples R China & 217 & 2624 & 24 & 12.09 & 1382323332 & 0.16 & 1.9 \\
\hline 8 & Sweden & 182 & 3899 & 23 & 21.42 & 9851852 & 18.47 & 395.76 \\
\hline 9 & Taiwan & 181 & 1724 & 19 & 9.52 & 23395600 & 7.74 & 73.69 \\
\hline 10 & Canada & 143 & 1419 & 17 & 9.92 & 36286378 & 3.94 & 39.11 \\
\hline 11 & Japan & 141 & 927 & 15 & 6.57 & 126323715 & 1.12 & 7.34 \\
\hline 12 & Netherlands & 131 & 1652 & 18 & 12.61 & 16979729 & 7.72 & 97.29 \\
\hline 13 & Australia & 106 & 2573 & 24 & 24.27 & 24309330 & 4.36 & 105.84 \\
\hline 14 & India & 105 & 1245 & 19 & 11.86 & 1326801576 & 0.08 & 0.94 \\
\hline 15 & Greece & 101 & 1213 & 19 & 12.01 & 10919459 & 9.25 & 111.09 \\
\hline 16 & Finland & 91 & 2034 & 17 & 22.35 & 5523904 & 16.47 & 368.22 \\
\hline 17 & Turkey & 90 & 1216 & 18 & 13.51 & 79622062 & 1.13 & 15.27 \\
\hline 18 & Belgium & 86 & 1085 & 16 & 12.62 & 11371928 & 7.56 & 95.41 \\
\hline 19 & Brazil & 79 & 1055 & 18 & 13.35 & 209567920 & 0.38 & 5.03 \\
\hline 20 & Denmark & 74 & 949 & 17 & 12.82 & 5690750 & 13 & 166.76 \\
\hline 21 & South Korea & 74 & 696 & 16 & 9.41 & 50503933 & 1.47 & 13.78 \\
\hline 22 & Iran & 64 & 817 & 15 & 12.77 & 80280000 & 0.8 & 10.18 \\
\hline 23 & Portugal & 61 & 625 & 14 & 10.25 & 10304434 & 5.92 & 60.65 \\
\hline 24 & Switzerland & 56 & 765 & 15 & 13.66 & 8379477 & 6.68 & 91.29 \\
\hline 25 & Austria & 49 & 818 & 16 & 16.69 & 8747000 & 5.6 & 93.52 \\
\hline 26 & New Zealand & 48 & 941 & 16 & 19.6 & 4565185 & 10.51 & 206.13 \\
\hline 27 & Singapore & 44 & 1281 & 17 & 29.11 & 5696506 & 7.72 & 224.87 \\
\hline 28 & Poland & 41 & 649 & 13 & 15.83 & 38593161 & 1.06 & 16.82 \\
\hline 29 & Czech Republic & 38 & 238 & 9 & 6.26 & 10560000 & 3.6 & 22.54 \\
\hline 30 & Israel & 34 & 316 & 9 & 9.29 & 8192463 & 4.15 & 38.57 \\
\hline 31 & Pakistan & 32 & 473 & 11 & 14.78 & 193200000 & 0.17 & 2.45 \\
\hline 32 & Slovenia & 32 & 351 & 11 & 10.97 & 2065000 & 15.5 & 169.98 \\
\hline 33 & Mexico & 29 & 208 & 8 & 7.17 & 128632004 & 0.23 & 1.62 \\
\hline 34 & Norway & 28 & 213 & 6 & 7.61 & 5271958 & 5.31 & 40.4 \\
\hline 35 & Malaysia & 27 & 429 & 13 & 15.89 & 30751602 & 0.88 & 13.95 \\
\hline 36 & Hungary & 27 & 392 & 12 & 14.52 & 9818000 & 2.75 & 39.93 \\
\hline 37 & Saudi Arabia & 26 & 308 & 9 & 11.85 & 32157974 & 0.81 & 9.58 \\
\hline 38 & Ireland & 21 & 191 & 7 & 9.1 & 4713993 & 4.45 & 40.52 \\
\hline 39 & Lithuania & 21 & 115 & 4 & 5.48 & 2872000 & 7.31 & 40.04 \\
\hline 40 & Serbia & 19 & 154 & 8 & 8.11 & 7057000 & 2.69 & 21.82 \\
\hline 41 & Kuwait & 17 & 47 & 4 & 2.76 & 4053000 & 4.19 & 11.6 \\
\hline 42 & Egypt & 15 & 253 & 10 & 16.87 & 93383574 & 0.16 & 2.71 \\
\hline 43 & Colombia & 13 & 125 & 5 & 9.62 & 48650000 & 0.27 & 2.57 \\
\hline 44 & Algeria & 11 & 240 & 9 & 21.82 & 40650000 & 0.27 & 5.9 \\
\hline 45 & Slovakia & 11 & 55 & 4 & 5 & 5429000 & 2.03 & 10.13 \\
\hline 46 & Cyprus & 10 & 175 & 7 & 17.5 & 1170000 & 8.55 & 149.57 \\
\hline 47 & Thailand & 9 & 449 & 4 & 49.89 & 68860000 & 0.13 & 6.52 \\
\hline 48 & Russia & 9 & 63 & 4 & 7 & 143439832 & 0.06 & 0.44 \\
\hline 49 & Romania & 9 & 53 & 5 & 5.89 & 19710000 & 0.46 & 2.69 \\
\hline 50 & Argentina & 8 & 72 & 5 & 9 & 43847277 & 0.18 & 1.64 \\
\hline
\end{tabular}


Table 9. Annual number of papers classified by countries

\begin{tabular}{|c|c|c|c|c|c|c|c|c|c|c|c|c|c|c|c|c|c|}
\hline $\mathrm{R}$ & Country & D1 & D2 & D3 & D4 & D5 & Total & 2017 & 2016 & 2015 & 2014 & 2013 & 2012 & 2011 & 2010 & 2009 & 2008 \\
\hline 1 & USA & 121 & 254 & 249 & 187 & 287 & 1098 & 34 & 33 & 19 & 26 & 46 & 35 & 23 & 27 & 27 & 17 \\
\hline 2 & United Kingdom & 14 & 25 & 100 & 63 & 131 & 333 & 19 & 14 & 7 & 18 & 20 & 23 & 13 & 8 & 4 & 5 \\
\hline 3 & Italy & 4 & 20 & 80 & 73 & 128 & 305 & 13 & 12 & 11 & 19 & 23 & 18 & 8 & 9 & 7 & 8 \\
\hline 4 & Germany & 15 & 33 & 91 & 72 & 85 & 296 & 5 & 11 & 5 & 7 & 12 & 17 & 7 & 8 & 9 & 4 \\
\hline 5 & Spain & 1 & 1 & 13 & 33 & 178 & 226 & 26 & 25 & 17 & 24 & 30 & 27 & 8 & 10 & 6 & 5 \\
\hline 6 & France & 6 & 25 & 54 & 52 & 88 & 225 & 7 & 10 & 5 & 9 & 14 & 12 & 6 & 12 & 2 & 11 \\
\hline 7 & Peoples R China & 0 & 3 & 7 & 35 & 172 & 217 & 38 & 36 & 16 & 19 & 12 & 15 & 7 & 7 & 12 & 10 \\
\hline 8 & Sweden & 19 & 25 & 73 & 35 & 30 & 182 & 4 & 2 & 1 & 4 & 3 & 4 & 4 & 3 & 3 & 2 \\
\hline 9 & Taiwan & 0 & 1 & 3 & 40 & 137 & 181 & 17 & 22 & 18 & 24 & 15 & 9 & 8 & 9 & 2 & 13 \\
\hline 10 & Canada & 24 & 28 & 22 & 14 & 55 & 143 & 9 & 6 & 3 & 4 & 9 & 8 & 8 & 2 & 1 & 5 \\
\hline 11 & Japan & 3 & 15 & 48 & 46 & 29 & 141 & 2 & 7 & 3 & 5 & 1 & 1 & 3 & 3 & 3 & 1 \\
\hline 12 & Netherlands & 3 & 33 & 54 & 20 & 21 & 131 & 1 & 3 & 2 & 0 & 3 & 2 & 3 & 1 & 3 & 3 \\
\hline 13 & Australia & 0 & 5 & 14 & 20 & 67 & 106 & 9 & 14 & 7 & 10 & 8 & 7 & 4 & 2 & 3 & 3 \\
\hline 14 & India & 1 & 8 & 8 & 8 & 80 & 105 & 22 & 28 & 7 & 6 & 5 & 3 & 2 & 2 & 2 & 3 \\
\hline 15 & Greece & 0 & 0 & 13 & 26 & 62 & 101 & 8 & 0 & 5 & 5 & 7 & 18 & 3 & 6 & 5 & 5 \\
\hline 16 & Finland & 0 & 3 & 38 & 35 & 15 & 91 & 1 & 0 & 0 & 1 & 1 & 2 & 3 & 1 & 2 & 4 \\
\hline 17 & Turkey & 0 & 0 & 1 & 19 & 70 & 90 & 10 & 14 & 4 & 10 & 10 & 6 & 5 & 3 & 4 & 4 \\
\hline 18 & Belgium & 4 & 9 & 20 & 15 & 38 & 86 & 3 & 3 & 1 & 3 & 6 & 4 & 8 & 5 & 1 & 4 \\
\hline 19 & Brazil & 0 & 0 & 4 & 14 & 61 & 79 & 11 & 15 & 4 & 5 & 9 & 4 & 3 & 4 & 1 & 5 \\
\hline 20 & South Korea & 0 & 0 & 0 & 16 & 58 & 74 & 13 & 11 & 4 & 7 & 1 & 10 & 2 & 2 & 4 & 4 \\
\hline 21 & Denmark & 2 & 9 & 24 & 17 & 22 & 74 & 0 & 2 & 0 & 0 & 3 & 1 & 6 & 3 & 2 & 5 \\
\hline 22 & Iran & 0 & 0 & 0 & 1 & 63 & 64 & 22 & 11 & 6 & 8 & 5 & 2 & 2 & 4 & 2 & 1 \\
\hline 23 & Portugal & 0 & 0 & 8 & 3 & 50 & 61 & 6 & 13 & 4 & 9 & 3 & 5 & 4 & 3 & 2 & 1 \\
\hline 24 & Switzerland & 6 & 6 & 12 & 14 & 18 & 56 & 2 & 2 & 1 & 2 & 1 & 1 & 3 & 1 & 2 & 3 \\
\hline 25 & Austria & 0 & 2 & 10 & 18 & 19 & 49 & 0 & 2 & 1 & 3 & 4 & 3 & 0 & 1 & 3 & 2 \\
\hline 26 & New Zealand & 0 & 2 & 3 & 14 & 29 & 48 & 0 & 1 & 0 & 5 & 4 & 1 & 10 & 2 & 1 & 5 \\
\hline 27 & Singapore & 0 & 0 & 1 & 13 & 30 & 44 & 2 & 4 & 1 & 4 & 8 & 7 & 1 & 1 & 1 & 1 \\
\hline 28 & Poland & 0 & 2 & 6 & 8 & 25 & 41 & 1 & 4 & 2 & 3 & 3 & 4 & 3 & 1 & 1 & 3 \\
\hline 29 & Czech Republic & 3 & 0 & 14 & 4 & 17 & 38 & 2 & 4 & 0 & 1 & 4 & 2 & 2 & 0 & 1 & 1 \\
\hline 30 & Israel & 5 & 9 & 11 & 6 & 3 & 34 & 0 & 1 & 0 & 0 & 0 & 0 & 1 & 0 & 1 & 0 \\
\hline 31 & Pakistan & 0 & 0 & 1 & 0 & 31 & 32 & 7 & 15 & 2 & 4 & 0 & 3 & 0 & 0 & 0 & 0 \\
\hline 32 & Slovenia & 0 & 0 & 1 & 17 & 14 & 32 & 1 & 0 & 3 & 2 & 2 & 0 & 1 & 1 & 3 & 1 \\
\hline 33 & Mexico & 0 & 0 & 3 & 4 & 22 & 29 & 3 & 8 & 0 & 2 & 4 & 1 & 1 & 1 & 2 & 0 \\
\hline 34 & Norway & 3 & 1 & 8 & 5 & 11 & 28 & 2 & 0 & 0 & 2 & 1 & 3 & 0 & 1 & 1 & 1 \\
\hline 35 & Malaysia & 0 & 0 & 0 & 2 & 25 & 27 & 3 & 5 & 2 & 4 & 4 & 4 & 2 & 0 & 1 & 0 \\
\hline 36 & Hungary & 0 & 4 & 5 & 7 & 11 & 27 & 1 & 2 & 0 & 1 & 2 & 1 & 3 & 0 & 1 & 0 \\
\hline 37 & Saudi Arabia & 3 & 1 & 4 & 7 & 11 & 26 & 2 & 6 & 0 & 2 & 0 & 1 & 0 & 0 & 0 & 0 \\
\hline 38 & Ireland & 0 & 1 & 10 & 1 & 9 & 21 & 0 & 1 & 0 & 0 & 1 & 3 & 3 & 0 & 1 & 0 \\
\hline 39 & Serbia & 0 & 0 & 2 & 1 & 16 & 19 & 2 & 1 & 2 & 5 & 0 & 2 & 2 & 1 & 1 & 0 \\
\hline 40 & Kuwait & 0 & 12 & 4 & 1 & 0 & 17 & 0 & 0 & 0 & 0 & 0 & 0 & 0 & 0 & 0 & 0 \\
\hline
\end{tabular}


Table 10 classifies publications based on the regions they originate from. In terms of the overall number of papers, North America ranks first followed by Europe, Asia and Oceania. Although, the number of papers from Oceania is lower compared to other regions, the per capita numbers for papers and citations are higher. The per capita citations received for publications from Oceania is 121.7 , which is by far the highest among all of the regions indicating high publication quality/relevance.

Table 10. Publication structure classified by supranational regions

\begin{tabular}{|c|c|c|c|c|c|c|c|c|}
\hline $\mathrm{R}$ & Region & TP & TC & $\mathrm{H}$ & $\mathrm{C} / \mathrm{P}$ & Population & P/Pop & C/Pop \\
\hline 1 & North America & 1241 & 18997 & 62 & 15.31 & 360405165 & 3.44 & 52.71 \\
\hline \multirow[t]{3}{*}{2} & Europe & 2533 & 35414 & 393 & 13.98 & 599570795 & 4.22 & 59.07 \\
\hline & Western and North Europe & 2132 & 29582 & 292 & 13.88 & 411599209 & 5.18 & 71.87 \\
\hline & Eastern Europe & 401 & 5832 & 101 & 14.54 & 187971586 & 2.13 & 31.03 \\
\hline \multirow[t]{4}{*}{3} & Asia & 1033 & 12163 & 205 & 11.77 & 3651581397 & 0.28 & 3.33 \\
\hline & Eastern Asia & 622 & 6034 & 78 & 9.7 & 1725986412 & 0.36 & 3.5 \\
\hline & Western Asia & 92 & 966 & 32 & 10.5 & 55029437 & 1.67 & 17.55 \\
\hline & South Asia & 319 & 5163 & 95 & 16.18 & 1870565548 & 0.17 & 2.76 \\
\hline 4 & Oceania & 154 & 3514 & 40 & 22.82 & 28874515 & 5.33 & 121.7 \\
\hline 5 & Latin and South America & 135 & 1512 & 40 & 11.2 & 448829051 & 0.3 & 3.37 \\
\hline 6 & Africa & 26 & 493 & 19 & 18.96 & 134033574 & 0.19 & 3.68 \\
\hline
\end{tabular}

Abbreviations available in previous tables except: $\mathrm{P} / \mathrm{Po}$ and $\mathrm{C} / \mathrm{Po}=$ Papers and cites per million inhabitants.

Table 11 presents an interesting analysis of the publications citing CMPB papers in terms of citing authors, universities, countries and journals. The top most citing authors are Mats O. Karlsson followed by Nick C. Fox, James Geoffrey Chase and U. Rajendra. It can be seen that the top citing authors also appear in the top 50 list of contributors to the CMPB (see Table 4). The top 3 most citing universities include INSERM France, Harvard University and Uppsala University. The highest number of citations for CMPB originated from the USA followed by the UK and PR China. In terms of journals, CMPB tops the list. It is not un-common for a journal to have self-cited papers as most of the papers are from the same discipline/research areas of biomedical research. Other source journals for citations include Plos One, Computers in Biology and Medicine, IEEE Transactions on Biomedical Engineering, and Physics in Medicine and Biology. All of these journals are top journals in the field of biomedical sciences indicating that CMPB is well regarded and has an impact in the research community. 
Table 11. Citing articles of CMPB: Authors, universities, countries and journals

\begin{tabular}{|c|c|c|c|c|c|c|c|c|}
\hline $\mathrm{R}$ & Author & TP & University & TP & Country & TP & Journal & TP \\
\hline 1 & Karlsson MO & 168 & INSERM France & 733 & USA & 12016 & Computer Methods Progr Biomed & 1317 \\
\hline 2 & Fox NC & 126 & Harvard U & 634 & UK & 3522 & Plos One & 506 \\
\hline 3 & Chase JG & 117 & Uppsala U & 586 & PR China & 2888 & Computers in Biology and Medicine & 382 \\
\hline 4 & Acharya UR & 116 & CNRS France & 534 & Germany & 2859 & IEEE Trans Biomedical Engineering & 314 \\
\hline 5 & Saxena PR & 96 & U College London & 507 & France & 2323 & Physics in Medicine and Biology & 294 \\
\hline 6 & Suri JS & 89 & Johns Hopkins U & 479 & Italy & 2311 & Antimicrobial Agents and Chemotherapy & 264 \\
\hline 7 & Slimani N & 86 & U Toronto & 475 & Canada & 1985 & J Biomedical Optics & 246 \\
\hline 8 & Ourselin S & 83 & Erasmus U Rotterdam & 467 & Netherlands & 1862 & Medical Physics & 237 \\
\hline 9 & Shaw GM & 83 & Assistance Publ Hop Paris & 436 & Sweden & 1713 & Expert Systems with Applications & 217 \\
\hline 10 & Bergman RN & 81 & Helmholtz Association & 430 & Spain & 1644 & Methods of information in Medicine & 206 \\
\hline 11 & Boeing $\mathrm{H}$ & 80 & Imperial College London & 404 & Australia & 1422 & British J Clinical Pharmacology & 199 \\
\hline 12 & Egorin MJ & 77 & Karolinska Institutet & 404 & India & 1104 & Biomedical Signal Processing and Control & 188 \\
\hline 13 & Jusko WJ & 77 & Utrecht U & 347 & Japan & 999 & J Medical Systems & 185 \\
\hline 14 & Riboli E & 76 & U Washington Seattle & 338 & Belgium & 903 & Medical Biological Engineering Computing & 182 \\
\hline 15 & Haffner SM & 73 & U Pennsylvania & 314 & Switzerland & 854 & J American Medical Informatics Assoc & 173 \\
\hline 16 & Overvad K & 71 & Lund $\mathrm{U}$ & 308 & South Korea & 789 & Neuroimage & 172 \\
\hline 17 & Ljungberg $\mathrm{M}$ & 70 & U Oxford & 307 & Brazil & 756 & Statistics in Medicine & 172 \\
\hline 18 & Pacini G & 69 & U Copenhagen & 300 & Taiwan & 723 & Int $\mathrm{J}$ Medical informatics & 159 \\
\hline 19 & Tjonneland A & 68 & CNR Italy & 293 & Denmark & 685 & J Clinical Pharmacology & 153 \\
\hline 20 & Danhof M & 64 & U California Los Angeles & 293 & Finland & 583 & $\mathrm{~J}$ Pharmacology and Experimental Therapeut & 150 \\
\hline 21 & Trichopoulou A & 59 & U Minnesota Twin Cities & 292 & Iran & 539 & IEEE Transactions on Medical Imaging & 148 \\
\hline 22 & Rossor MN & 58 & U Pittsburgh & 289 & Turkey & 513 & Artificial Intelligence in Medicine & 145 \\
\hline 23 & Forrest A & 56 & U Southern California & 283 & Austria & 512 & Physiological Measurement & 138 \\
\hline 24 & Viceconti M & 56 & Mcgill U & 280 & Greece & 477 & Cancer Chemotherapy and Pharmacology & 130 \\
\hline 25 & Grafman J & 55 & U Cambridge & 268 & Poland & 468 & J Pharmacokinetics and Pharmacodynamics & 130 \\
\hline 26 & & & U North Carolina Chapel & & & & & \\
\hline \multirow{3}{*}{27} & Mori S & 54 & Hill & 267 & Singapore & 417 & Clinical Pharmacology Therapeutics & 129 \\
\hline & & & & & New & & & \\
\hline & Urien S & 54 & German Cancer Res Center & 266 & Zealand & 375 & Sensors & 129 \\
\hline 28 & Beijnen JH & 53 & Duke U & 263 & Norway & 371 & J Biological Chemistry & 128 \\
\hline 29 & Tumino R & 52 & Stanford U & 262 & Malaysia & 342 & Clinical Pharmacokinetics & 127 \\
\hline 30 & Bueno-De-Mesquita HB & 51 & Leiden U & 249 & Portugal & 335 & Scientific Reports & 127 \\
\hline
\end{tabular}

\section{Mapping CMPB with VOS viewer software}

In the previous sections, we have provided a general overview with respect to the most relevant variables of the journal's performance. In addition to that, we will now provide an in-depth analysis of the journals citation structure and the development of the bibliographic connections over time. For the visualisation of these results, we use the VOS viewer software [1]. This allows us to create graphical maps to shed light on bibliographic coupling, citation and co-citation analysis, co-authorship, and co- occurrence of author keywords [17].

Firstly, Figure 2 provides an overview of the co-citations landscape for articles published in CMBP. Co-citation of journals occurs when two documents from two different journals receive a citation from the same document from a third journal. The graph visualizes the two journals that have received the citation. This will count as one co-citation link. Results are presented with a threshold of 50 co-citations. Connections are displayed with a threshold of 100 co-citations. The colours of the circles indicate the thematic clusters, to which the journals belong. 
While self-citations from CMBP unsurprisingly play the biggest role, strong connections can also be identified to IEEE Transactions on Biomedical Engineering and the IEEE Transactions on Medical Imaging. Also noteworthy are the strong connections with journals such as Biometrics, Journal of Applied Physiology and Circulation. This confirms CMBP's broad, interdisciplinary profile, citing also journals from outside the field.

To provide more insights into the development of co-citations over time and to understand the evolutions of each journal's influence, Figures 3-5 show snapshot of the cocitations landscape for the last three decades (slightly varying the connection thresholds). In the eighties and nineties, the number of journals as well as the overall number of papers published was smaller than today. Therefore, the graphical map is less dense than the ones representing the more recent decades (despite having set lower connection thresholds). Between 1988-1997, journals such as Am J Physiol, Comput Biomed Res, IEEE T Bio-Med Eng, Biometrics, and Diabetes Care were co-cited with CMPB. From the later nineties (Figure 4), strong connections to the fields of IEEE T Bio-Med Eng, IEEE T Med Imaging, IEEE T Pattern Anal, Biometrics, and J Biomech emerge, which are still seen today.

In the last decade, a similar co-citation structure was found (see Figure 5) with new journal additions such as Lect Notes Comput Sc, Comput Biol Med, Neuroimage and Expert Syst Appl. Top journals such as Lancet and Science are also featured in the top 50 co-citation analysis of CMPB indicating the quality of work that is being published and its impact on academia. A more detailed breakdown of co-citations and trends is provided in Table 12. 


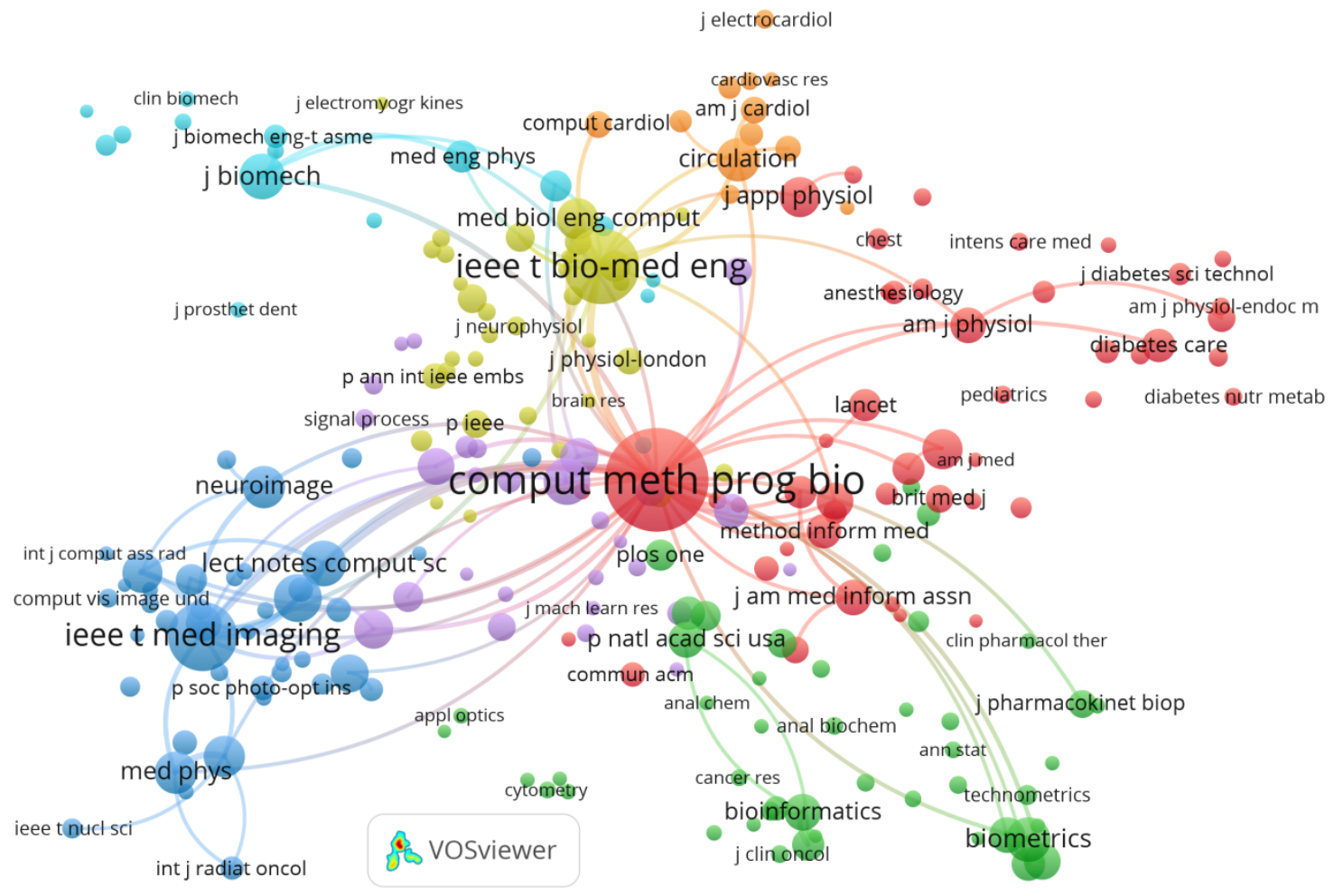

Figure 2. Co-citation of journals in CMPB: minimum citation threshold of 50 and 100 links 


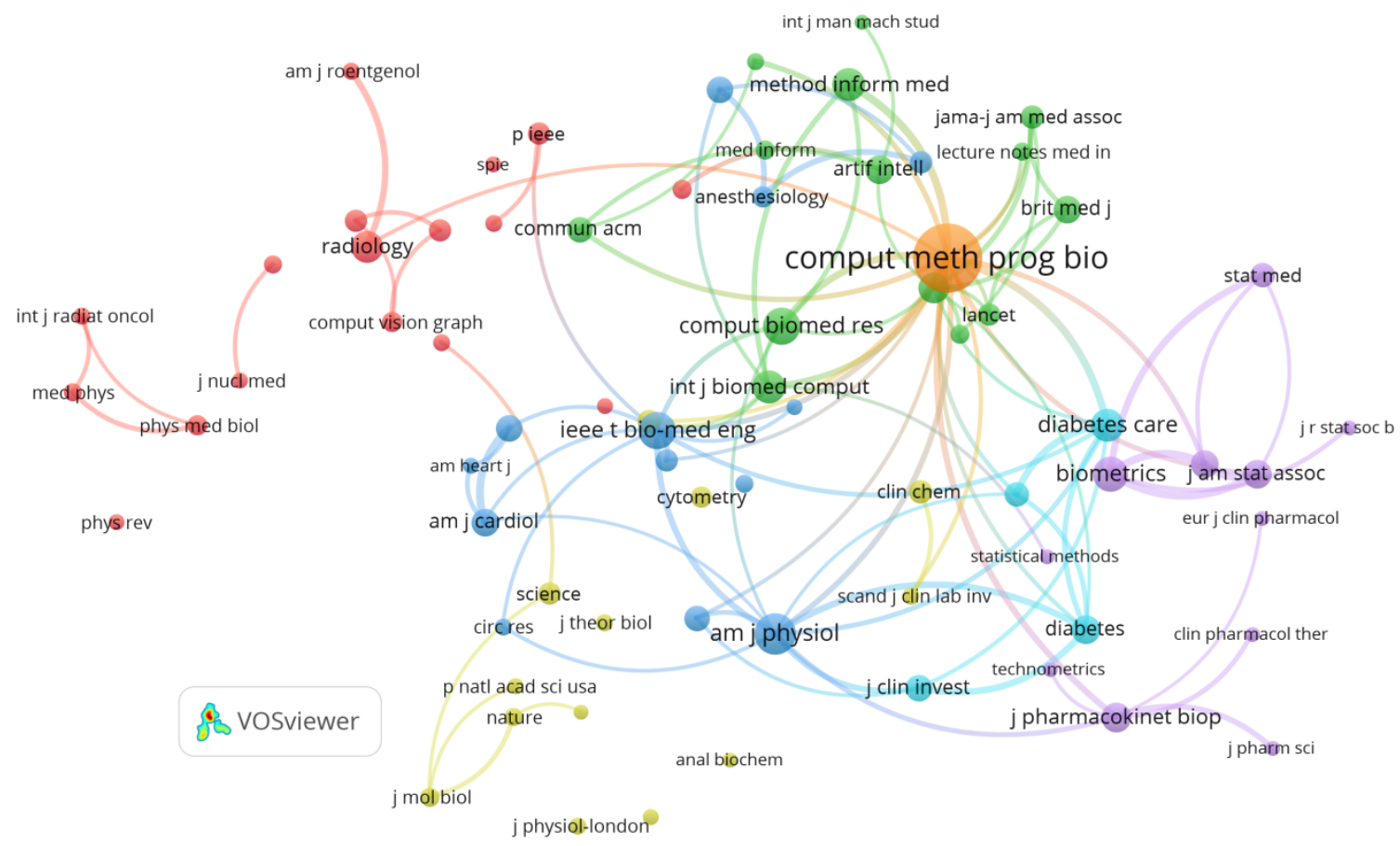

Figure 3. Co-citation of journals in CMPB: 1988-1997 (minimum citation threshold of 20 and 100 links)

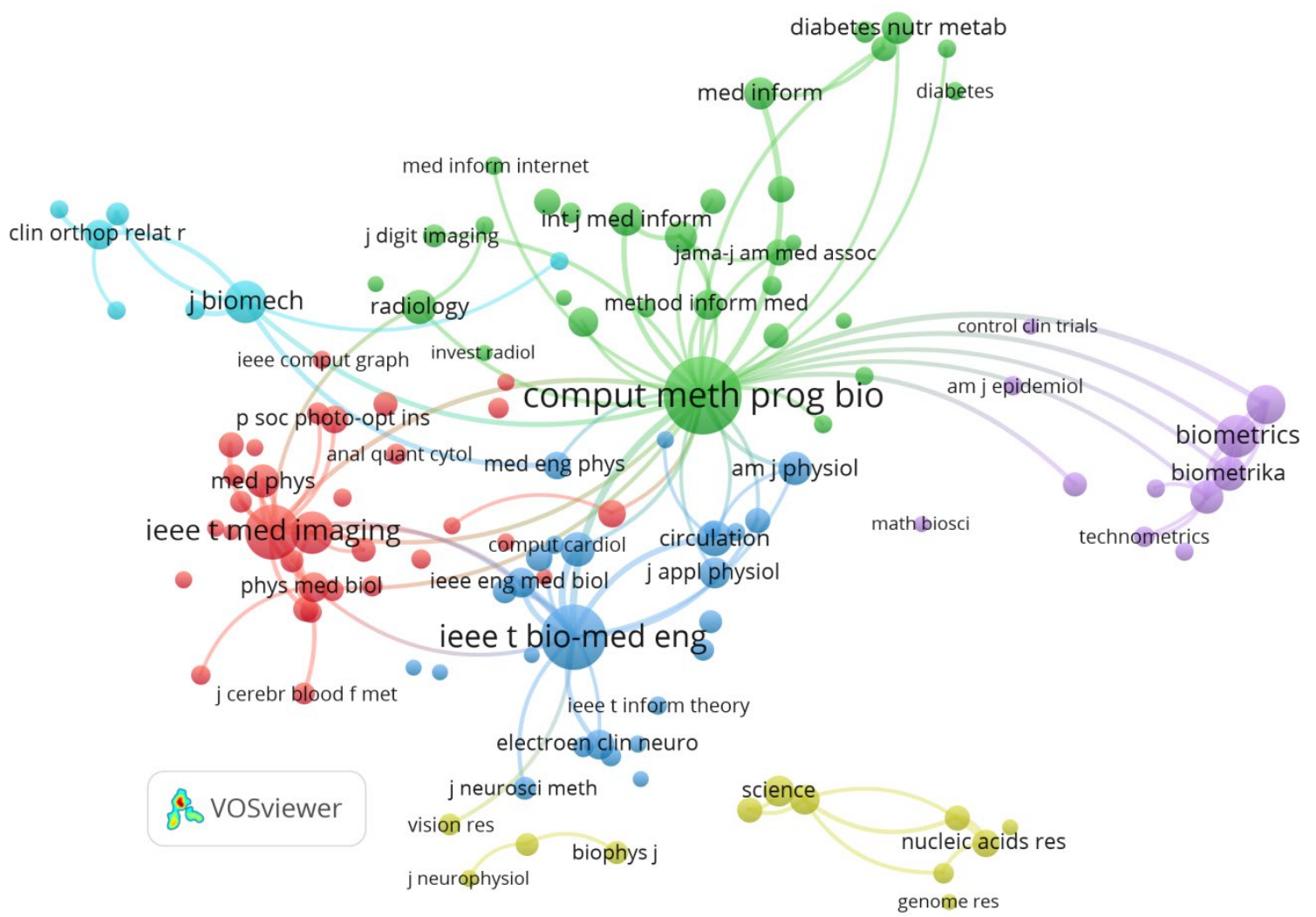

Figure 4. Co-citation of journals in CMPB: 1998-2007 (minimum citation threshold of 20 and 100 links) 


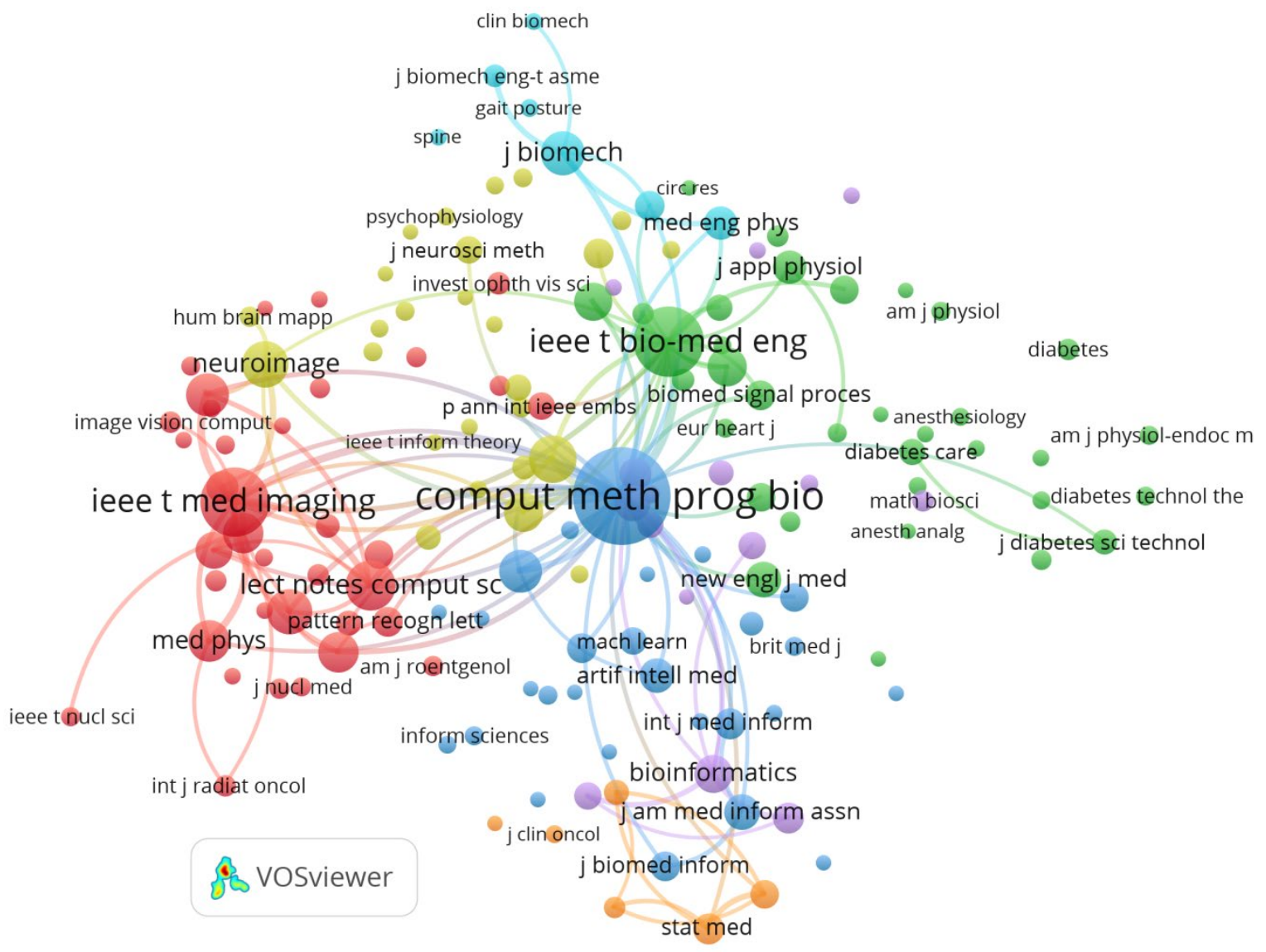

Figure 5. Co-citation of journals in CMPB: 2008-2017 (minimum citation threshold of 50 and 100 links) 
Table 12. Co-citation of journals in CMPB: Global and temporal analysis

\begin{tabular}{|c|c|c|c|c|c|c|c|c|c|c|c|c|}
\hline \multirow[b]{2}{*}{$\mathrm{R}$} & \multicolumn{3}{|c|}{ Global } & \multicolumn{3}{|c|}{$2008-2017$} & \multicolumn{3}{|c|}{$1998-2007$} & \multicolumn{3}{|c|}{ 1988-1997 } \\
\hline & Journal & Cit & CLS & Journal & Cit & CLS & Journal & Cit & CLS & Journal & Cit & CLS \\
\hline 1 & Comput Meth Prog Bio & 2835 & 2203.57 & Comput Meth Prog Bio & 1951 & 1536.66 & Comput Meth Prog Bio & 467 & 363.37 & Comput Meth Prog Bio & 398 & 224.03 \\
\hline 2 & IEEE T Bio-Med Eng & 1537 & 1251.74 & IEEE T Bio-Med Eng & 1019 & 835.4 & IEEE T Bio-Med Eng & 330 & 235.27 & Am J Physiol & 144 & 106.6 \\
\hline 3 & IEEE T Med Imaging & 1277 & 1036.91 & IEEE T Med Imaging & 995 & 805.04 & IEEE T Med Imaging & 237 & 175.79 & Comput Biomed Res & 118 & 102.22 \\
\hline 4 & IEEE T Pattern Anal & 619 & 561.94 & Lect Notes Comput Sc & 514 & 468.19 & IEEE T Pattern Anal & 144 & 126.99 & IEEE T Bio-Med Eng & 117 & 96.57 \\
\hline 5 & Comput Biol Med & 598 & 557.86 & Comput Biol Med & 468 & 439.91 & Biometrics & 139 & 101.43 & Biometrics & 103 & 74.3 \\
\hline 6 & J Biomech & 564 & 404.55 & Neuroimage & 447 & 334.03 & J Biomech & 138 & 91.04 & Diabetes Care & 96 & 69.8 \\
\hline 7 & Lect Notes Comput Sc & 561 & 515.05 & IEEE T Pattern Anal & 421 & 385.75 & Stat Med & 119 & 85.1 & Method Inform Med & 94 & 62.16 \\
\hline 8 & Biometrics & 525 & 394.03 & J Biomech & 401 & 272.09 & Circulation & 97 & 78.77 & Int J Biomed Comput & 91 & 74.01 \\
\hline 9 & Circulation & 521 & 453.48 & Expert Syst Appl & 388 & 338.9 & Med Biol Eng Comput & 96 & 85.15 & Radiology & 90 & 51.01 \\
\hline 10 & Neuroimage & 483 & 368.51 & Med Image Anal & 379 & 349.94 & Radiology & 94 & 70.27 & New Engl J Med & 84 & 68.79 \\
\hline 11 & Med Phys & 480 & 391.97 & Med Phys & 357 & 295.93 & Biometrika & 91 & 73.63 & J Pharmacokinet Biop & 76 & 53.81 \\
\hline 12 & Phys Med Biol & 470 & 376.76 & Phys Med Biol & 343 & 268.87 & Int J Med Inform & 89 & 72.29 & Artif Intell & 72 & 47.35 \\
\hline 13 & Med Biol Eng Comput & 458 & 428.69 & Pattern Recogn & 335 & 311.69 & Med Phys & 88 & 63.24 & Diabetes & 70 & 54.71 \\
\hline 14 & Med Image Anal & 430 & 397.59 & IEEE T Inf Technol B & 329 & 305.49 & Am J Physiol & 86 & 72.98 & J Am Stat Assoc & 69 & 55.17 \\
\hline 15 & New Engl J Med & 428 & 395.61 & Circulation & 324 & 287.7 & J Am Stat Assoc & 86 & 69.75 & Am J Cardiol & 67 & 43.66 \\
\hline 16 & J Appl Physiol & 425 & 290.72 & Bioinformatics & 312 & 255.05 & Med Inform & 81 & 64.51 & Biometrika & 66 & 52.07 \\
\hline 17 & Pattern Recogn & 408 & 377.36 & Med Biol Eng Comput & 296 & 279.51 & $\mathrm{~J}$ Am Med Inform Assn & 80 & 62.49 & Brit Med J & 66 & 51.55 \\
\hline 18 & Expert Syst Appl & 398 & 350.63 & IEEE T Image Process & 292 & 267.79 & Diabetes Nutr Metab & 79 & 35.43 & $\mathrm{~J}$ Clin Invest & 62 & 48.14 \\
\hline 19 & Radiology & 383 & 306.89 & PLOS One & 275 & 254.07 & Science & 77 & 68.75 & Circulation & 60 & 46.59 \\
\hline 20 & Stat Med & 378 & 286.67 & $\mathrm{~J}$ Am Med Inform Assn & 262 & 212.04 & J Appl Physiol & 74 & 43.27 & Electroen Clin Neuro & 59 & 32.33 \\
\hline 21 & Bioinformatics & 365 & 302.62 & New Engl J Med & 261 & 245.05 & Clin Orthop Relat R & 73 & 52.96 & J Appl Physiol & 57 & 35.25 \\
\hline 22 & IEEE T Inf Technol B & 364 & 340.39 & Artif Intell Med & 248 & 221.98 & Method Inform Med & 73 & 55.99 & Commun ACM & 55 & 40.39 \\
\hline 23 & Comput Biomed Res & 357 & 303.11 & J Appl Physiol & 237 & 162.77 & Phys Med Biol & 72 & 62.19 & Stat Med & 52 & 38.55 \\
\hline 24 & Am J Physiol & 354 & 297.54 & Med Eng Phys & 232 & 212.86 & P Natl Acad Sci Usa & 71 & 60.82 & Diabetologia & 50 & 43.18 \\
\hline 25 & $\mathrm{~J}$ Am Med Inform Assn & 350 & 286.07 & P Natl Acad Sci Usa & 223 & 201.62 & IEEE Eng Med Biol & 70 & 60.09 & Comput Biol Med & 47 & 37.05 \\
\hline 26 & J Am Stat Assoc & 349 & 299.17 & Comput Med Imag Grap & 217 & 196.69 & Electroen Clin Neuro & 69 & 51.3 & Clin Chem & 46 & 23.94 \\
\hline 27 & IEEE T Image Process & 341 & 315.08 & Stat Med & 204 & 143.92 & Artif Intell Med & 68 & 54.54 & JAMA-J Am Med Assoc & 46 & 38.66 \\
\hline 28 & P Natl Acad Sci Usa & 341 & 305.97 & Int J Med Inform & 199 & 168 & Comput Biol Med & 61 & 54.63 & Science & 45 & 38.08 \\
\hline 29 & Artif Intell Med & 331 & 294.46 & Nucleic Acids Res & 195 & 142.59 & Med Eng Phys & 60 & 54.57 & IEEE T Med Imaging & 44 & 32.56 \\
\hline 30 & Method Inform Med & 324 & 252.67 & J Biomed Inform & 191 & 174.16 & P Soc Photo-Opt Ins & 60 & 51.63 & Lancet & 44 & 39.88 \\
\hline 31 & Science & 314 & 291.59 & Pattern Recogn Lett & 191 & 184.55 & Nucleic Acids Res & 59 & 41.81 & Med Biol Eng Comput & 44 & 39.38 \\
\hline 32 & Biometrika & 302 & 254.72 & Ann Biomed Eng & 190 & 176.87 & J Telemed Telecare & 57 & 28.2 & P IEEE & 44 & 37.23 \\
\hline 33 & Diabetes Care & 298 & 247.64 & Biomed Signal Proces & 189 & 181.93 & New Engl J Med & 57 & 53.86 & Brit J Anaesth & 43 & 23.96 \\
\hline 34 & Med Eng Phys & 294 & 272.72 & Clin Neurophysiol & 186 & 157.06 & Ann Biomed Eng & 56 & 51.03 & IEEE T Pattern Anal & 43 & 31.01 \\
\hline 35 & JAMA-J Am Med Assoc & 292 & 263.09 & J Med Syst & 184 & 158.61 & JAMA-J Am Med Assoc & 56 & 49.13 & Anesthesiology & 39 & 32.8 \\
\hline 36 & Nucleic Acids Res & 292 & 212.28 & Lancet & 175 & 162.05 & Bioinformatics & 53 & 39.87 & Cytometry & 38 & 15.98 \\
\hline 37 & Int J Med Inform & 288 & 243.03 & Biometrics & 172 & 135.14 & Brit Med J & 51 & 46.21 & Phys Med Biol & 37 & 26.21 \\
\hline 38 & Lancet & 277 & 257.57 & Radiology & 171 & 155.65 & Comput Biomed Res & 51 & 47.55 & Comput Vision Graph & 34 & 27.92 \\
\hline 39 & PLOS One & 275 & 256.14 & JAMA-J Am Med Assoc & 170 & 156.54 & Lancet & 51 & 46.03 & Ann Intern Med & 33 & 28.59 \\
\hline 40 & Ann Biomed Eng & 272 & 253.62 & Physiol Meas & 168 & 152.53 & Med Image Anal & 51 & 43.59 & J Mol Biol & 33 & 19.52 \\
\hline
\end{tabular}

Abbreviations: $\mathrm{Cit}=$ Citations; $\mathrm{CLS}=$ Citation link strength. 


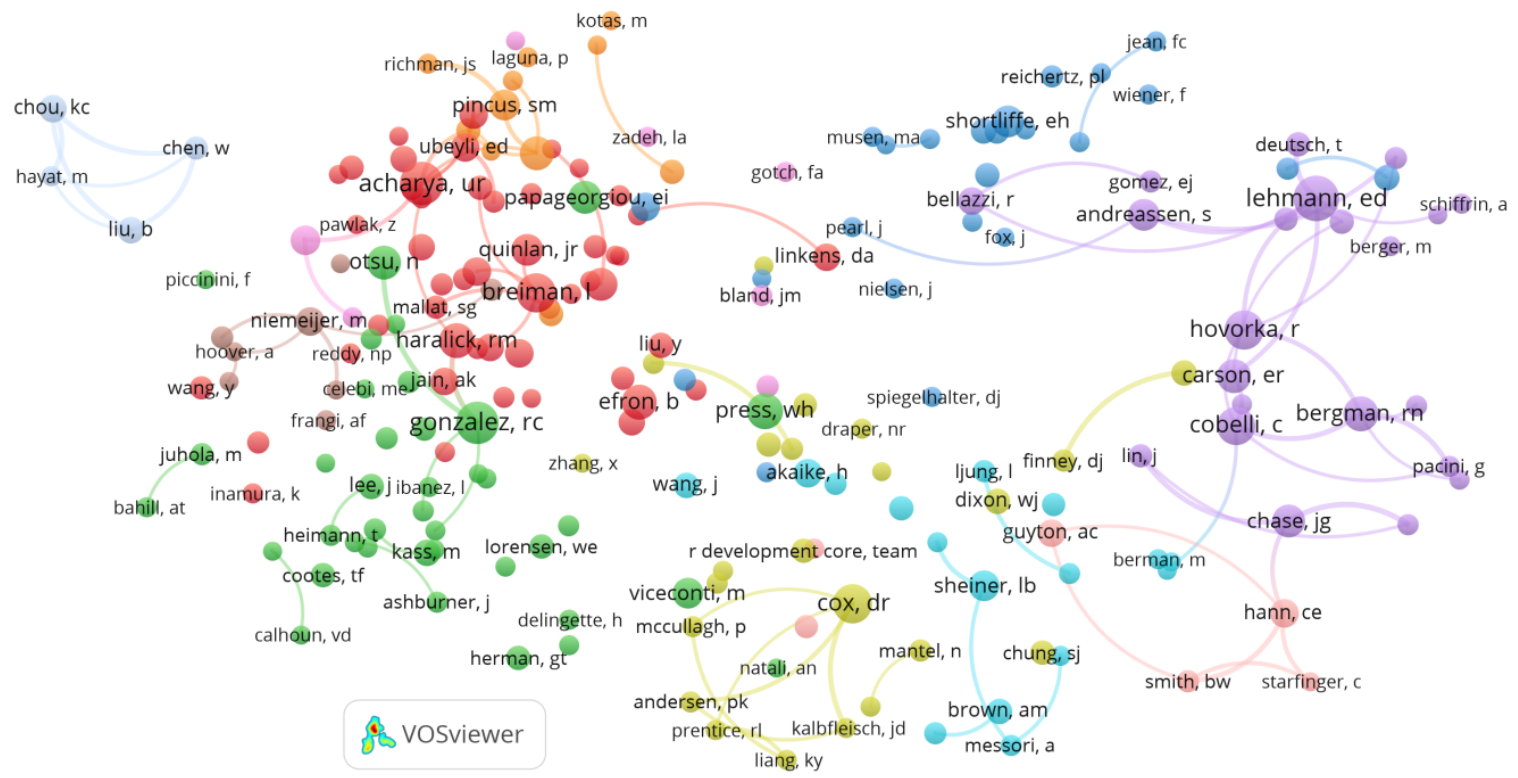

Figure 6. Co-citation of authors in CMPB: minimum citation threshold of 20 and 100 links

We will now take a look at the co-citation of authors who have published in CMBP. Similar to the co-citation of journals, a co-citation of authors occurs, when the authors of two documents cite the same third document [2]. CMBP's most productive authors are shown in Figure 6 using a citation threshold of 20 and bibliographic coupling links between authors of more than 100. Looking at the network of authors in Figure 6 and an analysis of the authors discipline areas, we could establish that authors having similar research domains cite similar bibliographic material. For example the cluster of authors including Acharya and Breiman work in signal processing analysis for heart disorders; similarly the cluster with Lehmann, Cobelli, Hovorka and Bergman work on decision support systems for Diabetes; and the cluster with Gonzales and Otsu work towards image processing and analysis. 


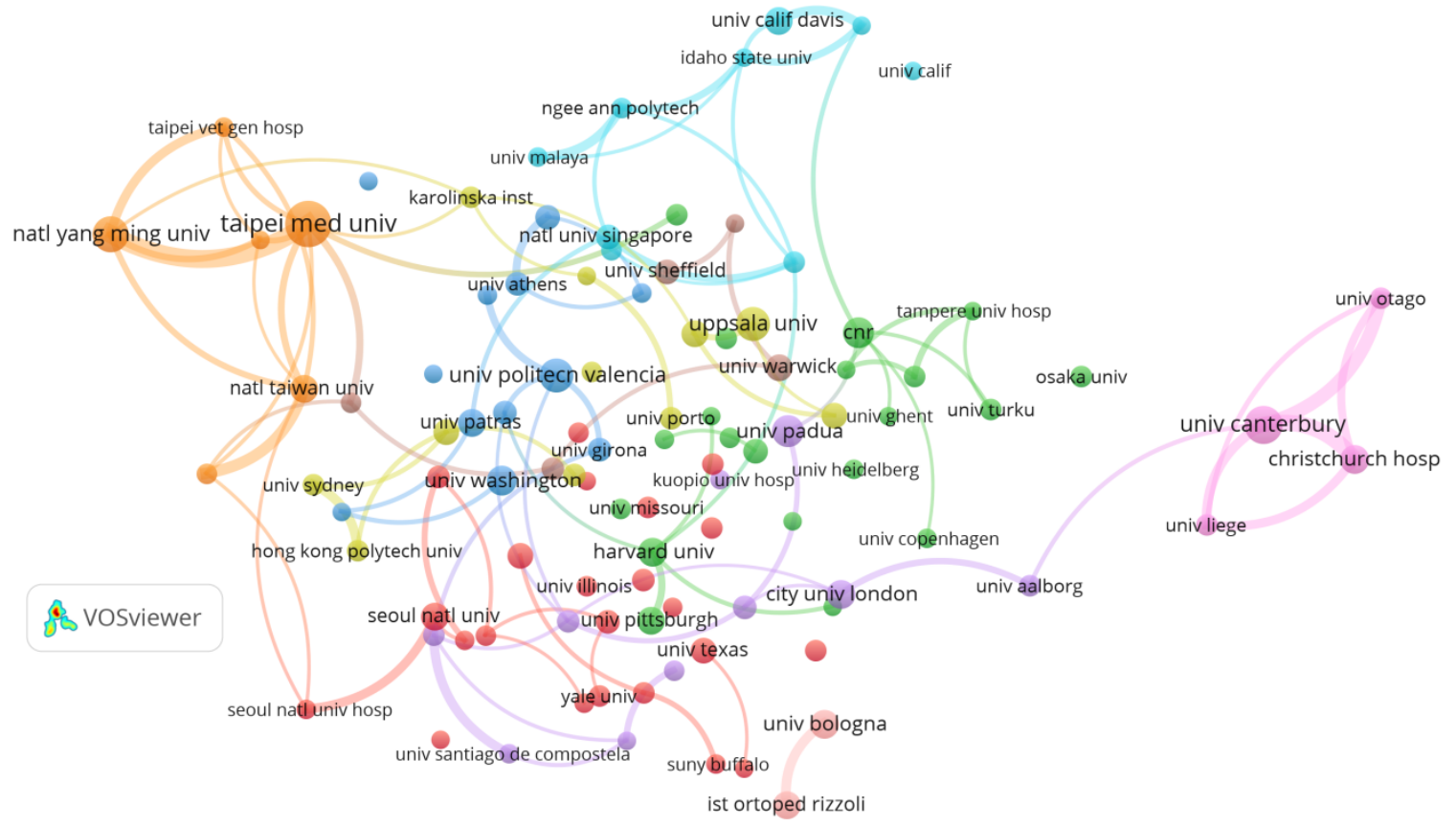

Figure 7. Bibliographic coupling of institutions publishing in CMPB: minimum publication threshold of 10 documents and 100 links

Moving on from this, we will look at the bibliographic coupling of institution publishing in CMBP in Figure 7. In line with previous analyses, thresholds are set for documents (10) and links (100). Results are in line with those diplayed in Table 6. An interesting result here is that the universities from the same country (or to a lesser extent the same continent) tend to have a similar profile between them, often clustered together and with strong connections. Figure 8 presents the bibliometric coupling between different countries. It shows that there are strong links between the USA and China, Spain, Germany, Taiwan and England. 


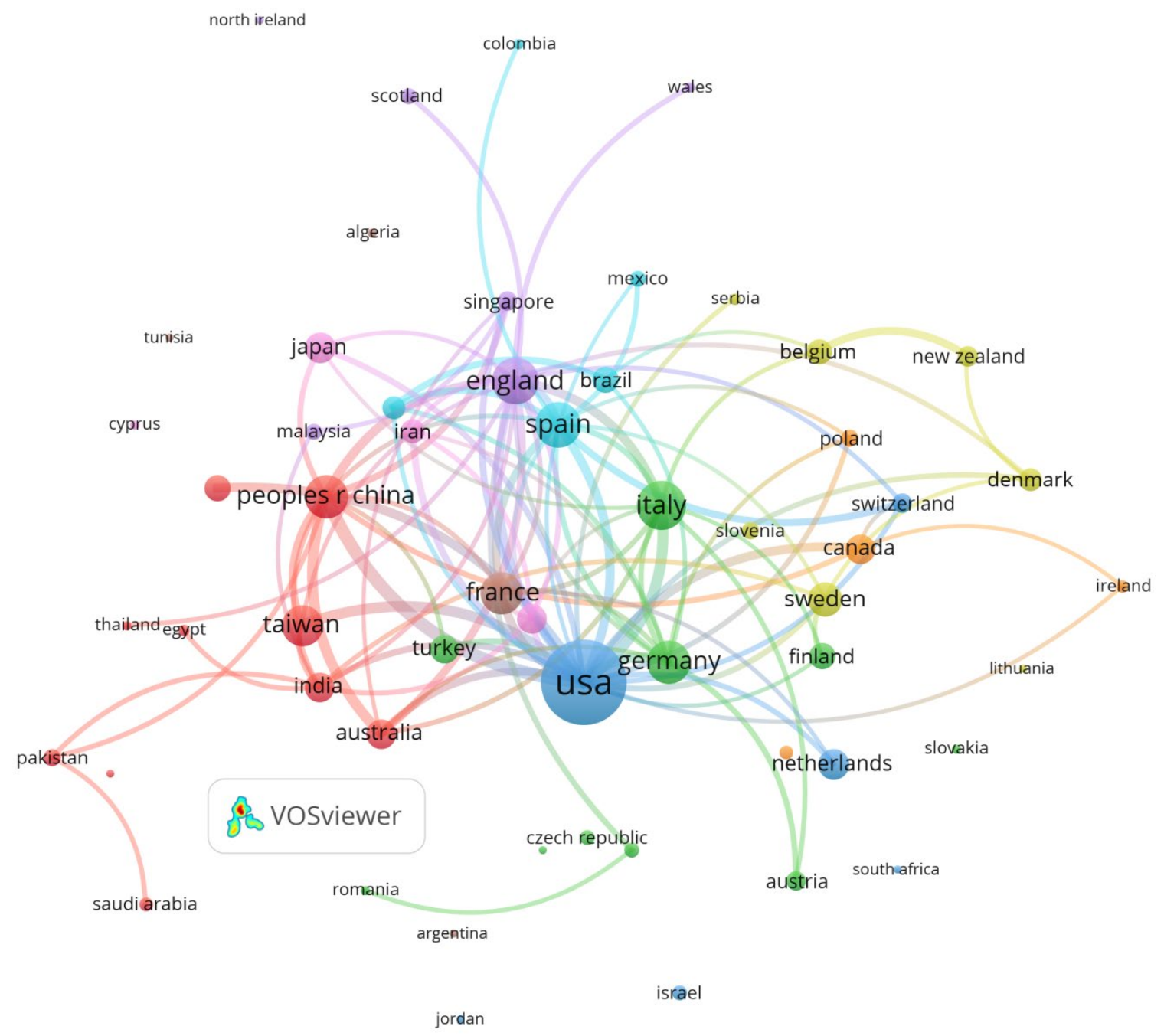

Figure 8. Bibliographic coupling of countries publishing in CMPB: minimum publication threshold of 5 documents and 50 links

Following on towards the keywords analysis from the articles published in CMPB, Figure 9 shows the network mapping between various keywords used by authors. As CMPB aims at publishing computer methods and programs applied to the biomedical research field, the keyword analyses reveal interesting co-relations between the biomedical area of research and the computer methods that were used. Strong clusters are identified around the keywords classification (including deep learning), PACS (picture archiving and communication system), heart rate variability, survival analysis and simulation. The cluster around classification includes areas where some sort of machine learning methods were developed/applied towards image processing. Other topics of research can be identified such as (i) signal processing in case of heart rate variability, (ii) simulation models for diabetes, 
(iii) use of PACS data for its use in health technology assessments and image processing, (iv) decision support systems development based on healthcare records and (v) survivability analysis for health conditions. All of these topics are popular among CMPB authors. When analysing the trends in keywords co-occurrences from 1988-2017, we see an interesting trend. In 1988-1997, keywords such as computer simulations, PACS, image processing, mathematical modelling, expert systems, knowledge-based systems, and decision support systems were common. In the period 1998-2007, machine learning related keywords such as segmentation, classification, neural networks were beginning to pick up. Simulation models and software development for a variety of health data analyses were prominent in this period as well. However, in the last decade (2008-2017), keywords related to the machine learning field such as classification, segmentation, feature selection/ extraction, image processing became the central theme in CMPB. This may be due to the advancements in the storage, retrieval and analysis of large health datasets in form of images, videos, text and numeric records during the last decade. On closer analysis of recent publications (in the last 2 years) in CMPB, we found that deep learning was an important research area. In particular, its application in healthcare areas where physiological signals are available. The application areas of deep learning included Electroencephalogram (EEG), Electrocardiogram (ECG) and Electrooculogram (EOG). It is highlighted through the publications that the deep learning approach performs better for large and varied datasets for machine-based classification problems. In the future, it is anticipated that there would be growing research on the application of deep learning in biomedical research fields. Table 13 provides a detailed account of keywords co-occurrences in CMPB articles. 


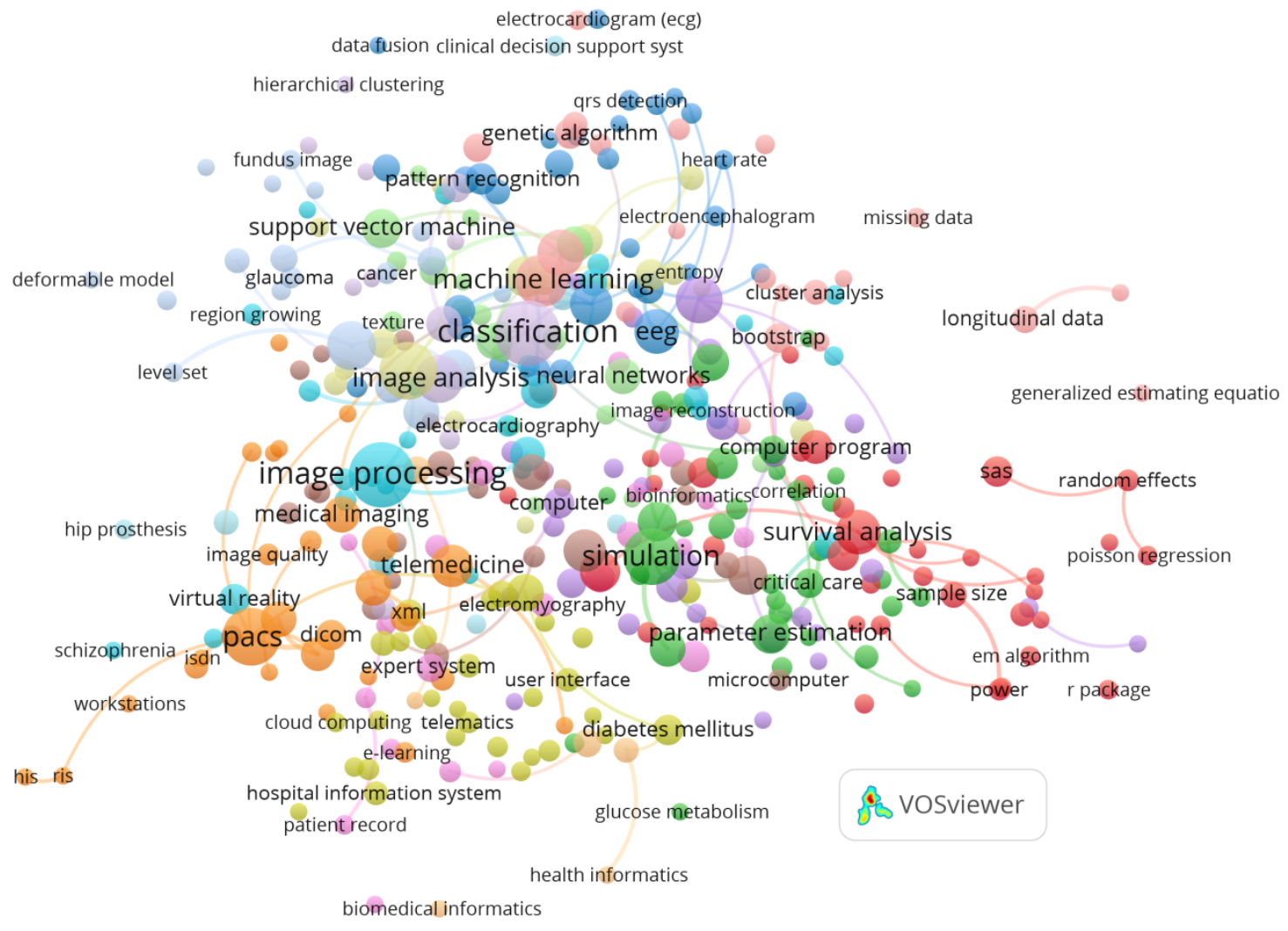

Figure 9. Co-occurrence of author keywords in CMPB: minimum occurrence threshold of 5 and 100 links 


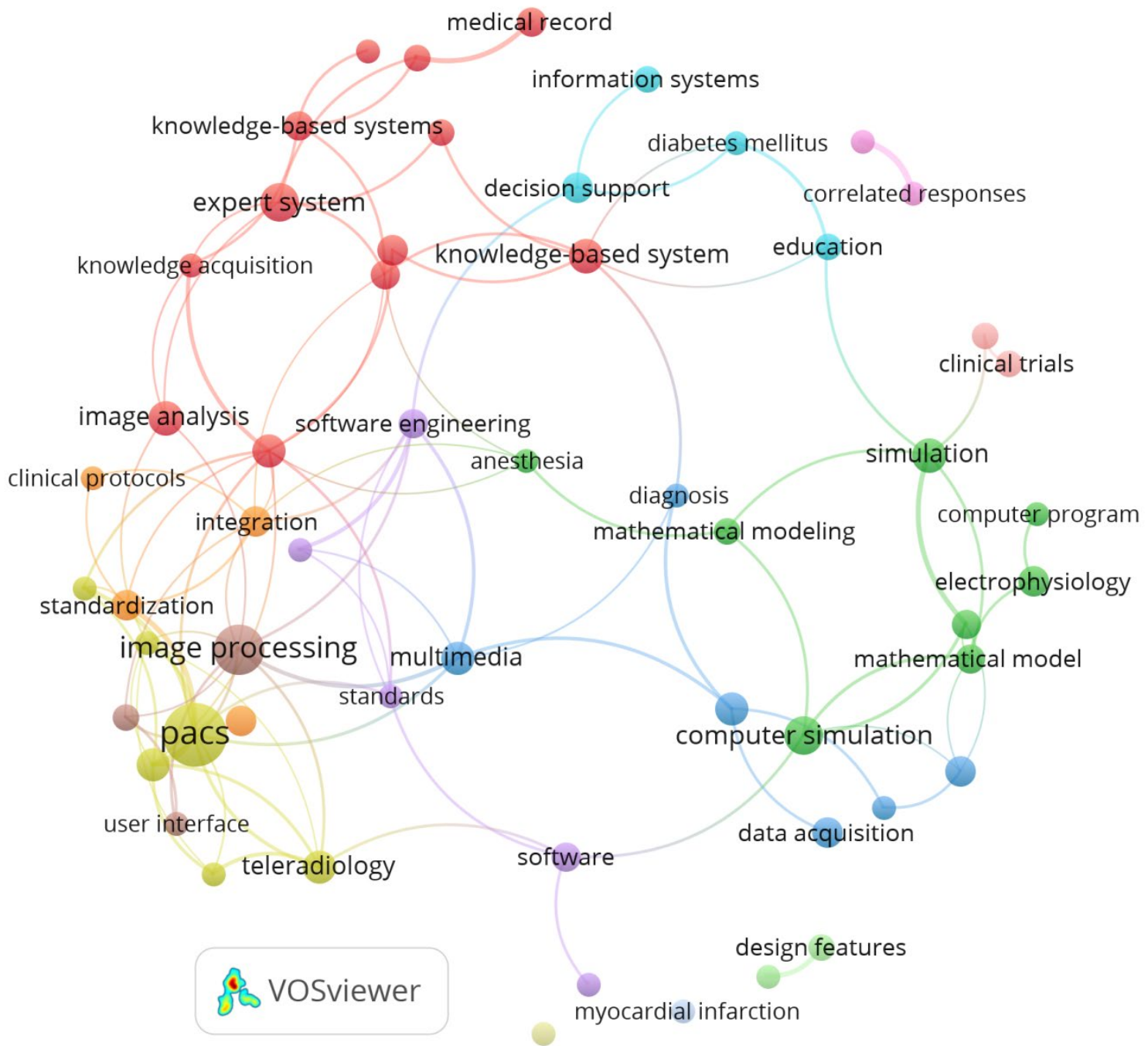

Figure 10. Co-occurrence of author keywords in CMPB: 1988-1997 (minimum occurrence threshold of 3 and 100 links) 


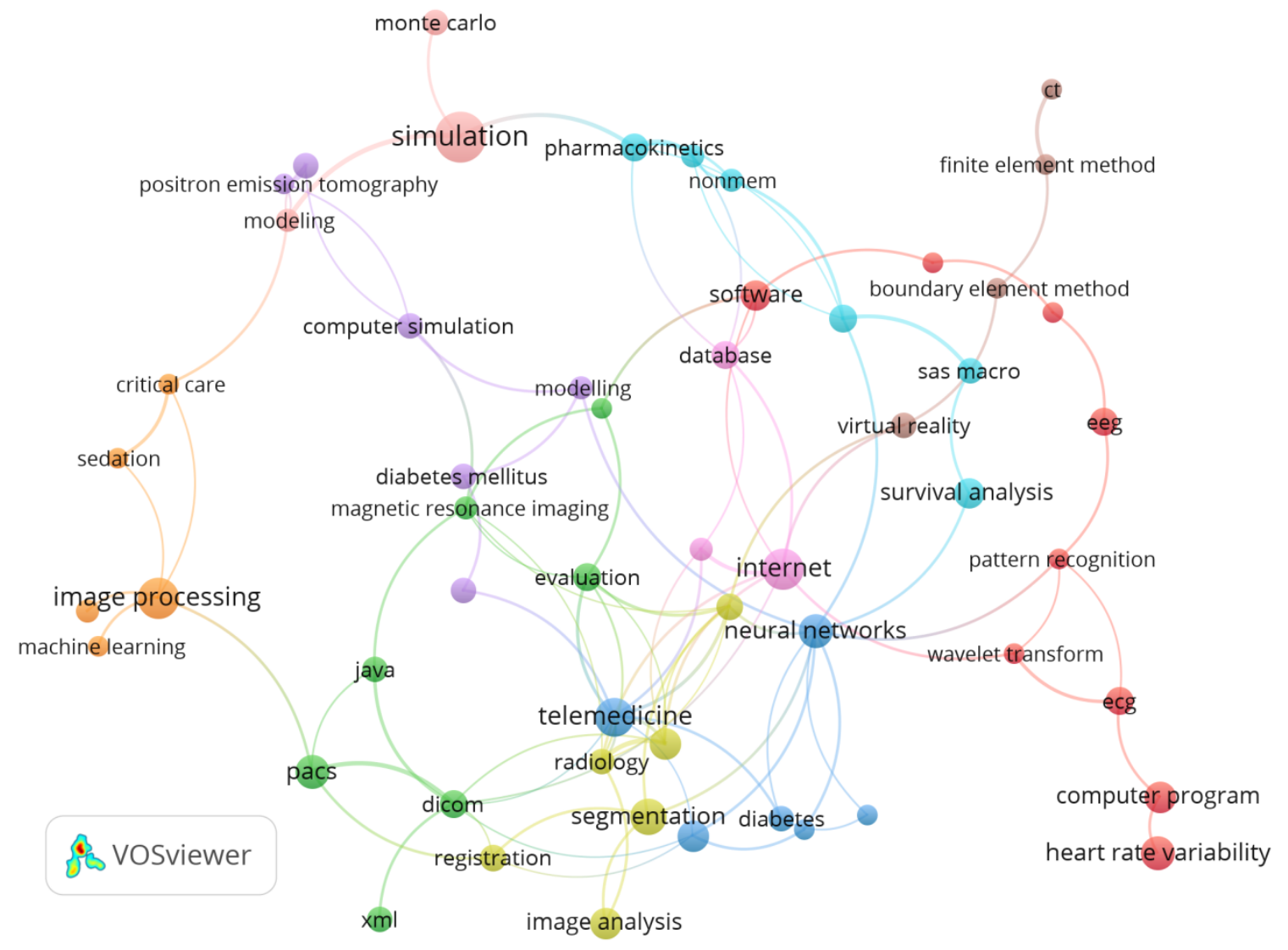

Figure 11. Co-occurrence of author keywords in CMPB: 1998-2007 (minimum occurrence threshold of 5 and 50 links) 


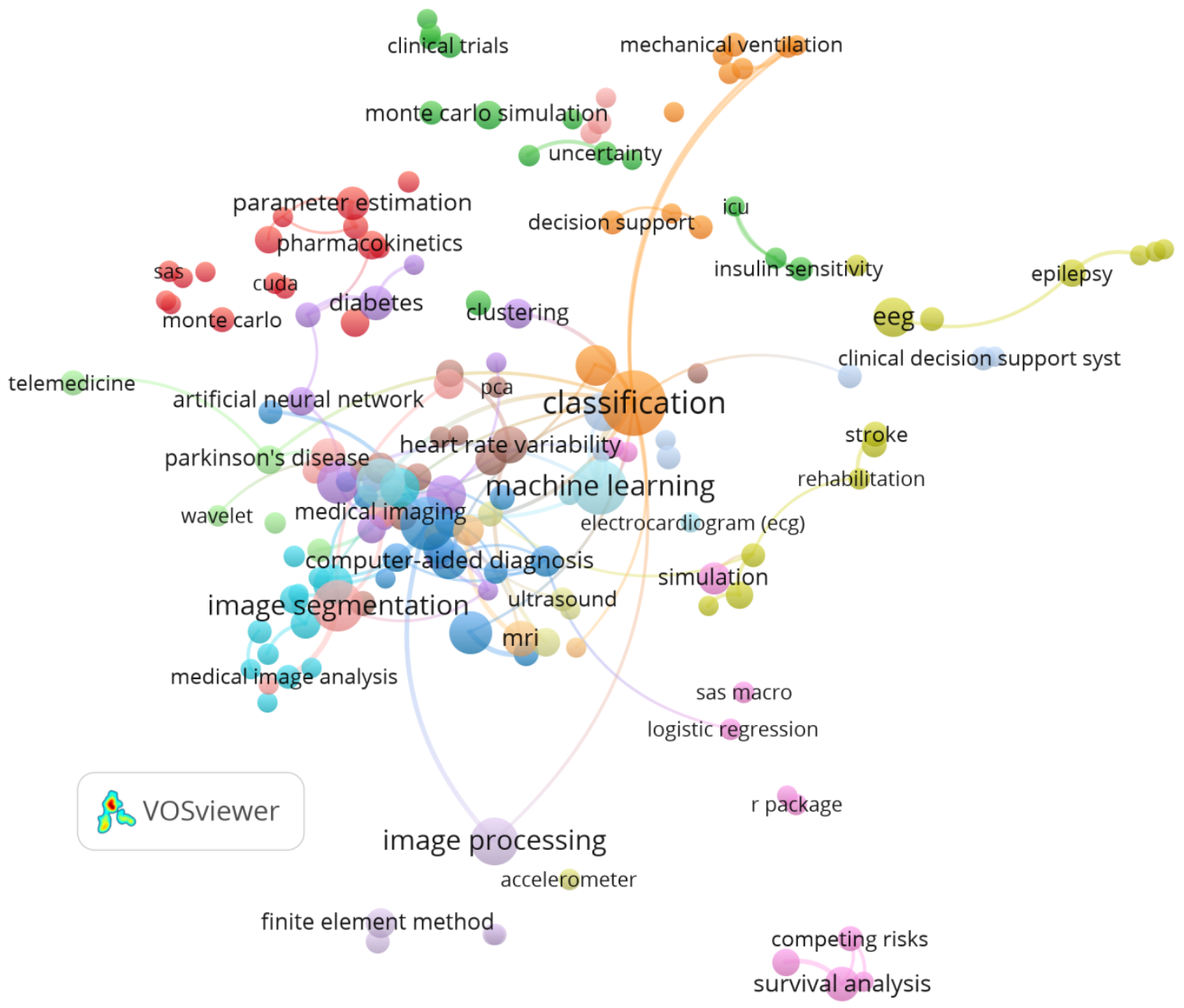

Figure 12. Co-occurrence of author keywords in CMPB: 2008-2017 (minimum occurrence threshold of 5 and 100 links) 
Table 13. Co-occurrence of author keywords in CMPB: Global and temporal analysis

\begin{tabular}{|c|c|c|c|c|c|c|c|c|c|c|c|c|}
\hline & \multicolumn{3}{|l|}{ Global } & \multicolumn{3}{|l|}{$2008-2017$} & \multicolumn{3}{|c|}{ 1998-2007 } & \multicolumn{3}{|l|}{ 1988-1997 } \\
\hline $\mathrm{R}$ & Keyword & Occ & Co-oc & Keyword & Occ & Co-oc & Keyword & Occ & Co-oc & Keyword & Occ & $\mathrm{Co}-\mathrm{oc}$ \\
\hline 1 & Image Processing & 68 & 43 & Classification & 52 & 39 & Simulation & 28 & 7 & PACS & 33 & 17 \\
\hline 2 & Classification & 67 & 49 & EEG & 39 & 18 & Image Processing & 19 & 4 & Image Processing & 21 & 8 \\
\hline 3 & EEG & 61 & 43 & Machine Learning & 37 & 27 & Internet & 19 & 8 & Knowledge-Based System & 17 & 9 \\
\hline 4 & Segmentation & 53 & 35 & Segmentation & 35 & 23 & EEG & 18 & 5 & Decision Support & 16 & 5 \\
\hline 5 & PACS & 52 & 35 & Image Segmentation & 32 & 20 & Telemedicine & 17 & 9 & Computer Simulation & 12 & 5 \\
\hline 6 & Simulation & 51 & 36 & Feature Selection & 29 & 17 & Segmentation & 15 & 4 & Expert System & 12 & 4 \\
\hline 7 & Machine Learning & 44 & 37 & Image Processing & 28 & 16 & Heart Rate Variability & 13 & 1 & Modeling & 11 & 9 \\
\hline 8 & Decision Support & 38 & 22 & Feature Extraction & 23 & 17 & Neural Networks & 13 & 9 & Image Analysis & 10 & 2 \\
\hline 9 & Image Segmentation & 38 & 27 & Support Vector Machine & 23 & 11 & PACS & 13 & 5 & Simulation & 10 & 7 \\
\hline 10 & Image Analysis & 36 & 28 & Artificial Neural Networks & 22 & 11 & Modeling & 12 & 9 & Computer & 9 & 5 \\
\hline 11 & Feature Selection & 34 & 23 & Breast Cancer & 19 & 14 & Classification & 11 & 3 & Evaluation & 9 & 8 \\
\hline 12 & Heart Rate Variability & 34 & 24 & Computed Tomography & 19 & 15 & Computer Program & 11 & 2 & Multimedia & 9 & 8 \\
\hline 13 & Modeling & 34 & 28 & Data Mining & 19 & 9 & Image Analysis & 11 & 2 & Radiology & 9 & 9 \\
\hline 14 & Survival Analysis & 31 & 21 & Magnetic Resonance Imaging & 19 & 13 & Teleradiology & 11 & 7 & Teleradiology & 9 & 7 \\
\hline 15 & Telemedicine & 29 & 21 & Computer-Aided Diagnosis & 17 & 13 & Decision Support & 10 & 4 & Data Acquisition & 8 & 1 \\
\hline 16 & Artificial Neural Networks & 29 & 19 & Heart Rate Variability & 17 & 10 & Software & 10 & 4 & Electrophysiology & 8 & 2 \\
\hline 17 & Computer Simulation & 28 & 13 & MRI & 16 & 11 & Survival Analysis & 10 & 2 & Integration & 8 & 5 \\
\hline 18 & Feature Extraction & 27 & 25 & Diabetes & 15 & 10 & Bootstrap & 9 & 5 & Microcomputer & 8 & 2 \\
\hline 19 & Magnetic Resonance Imaging & 26 & 21 & Image Analysis & 15 & 8 & Database & 9 & 3 & Standardization & 8 & 6 \\
\hline 20 & Software & 26 & 16 & Survival Analysis & 15 & 6 & Dicom & 9 & 7 & Telematics & 8 & 1 \\
\hline 21 & Parameter Estimation & 25 & 21 & Parameter Estimation & 14 & 9 & Evaluation & 9 & 5 & Intensive Care & 7 & 4 \\
\hline 22 & Diabetes & 24 & 16 & Simulation & 13 & 7 & Pharmacokinetics & 9 & 8 & Mathematical Model & 7 & 4 \\
\hline 23 & Pharmacokinetics & 24 & 18 & Atrial Fibrillation & 12 & 8 & Medical Imaging & 8 & 7 & Medical Record & 7 & 3 \\
\hline 24 & Support Vector Machine & 24 & 14 & Decision Support & 12 & 7 & Registration & 8 & 3 & Software & 7 & 4 \\
\hline 25 & Data Mining & 22 & 12 & Genetic Algorithm & 12 & 8 & Computer Simulation & 7 & 3 & Software Engineering & 7 & 7 \\
\hline 26 & Computed Tomography & 21 & 18 & Mutual Information & 12 & 8 & Diabetes & 7 & 2 & Artificial Intelligence & 6 & 2 \\
\hline 27 & Internet & 21 & 12 & SVM & 12 & 5 & Diabetes Mellitus & 7 & 2 & Clinical Trials & 6 & 1 \\
\hline 28 & Medical Imaging & 21 & 18 & Clustering & 11 & 8 & Java & 7 & 3 & Design Features & 6 & 3 \\
\hline 29 & Neural Networks & 21 & 17 & Diabetic Retinopathy & 11 & 9 & Monte Carlo & 7 & 1 & Education & 6 & 3 \\
\hline 30 & Breast Cancer & 20 & 15 & Finite Element Method & 11 & 3 & Parameter Estimation & 7 & 4 & Hospital Information System & 6 & 3 \\
\hline 31 & Computer-Aided Diagnosis & 20 & 17 & Image Registration & 11 & 7 & Radiology & 7 & 7 & Information Systems & 6 & 1 \\
\hline 32 & Database & 20 & 12 & Modelling & 11 & 9 & SAS Macro & 7 & 3 & Mathematical Modeling & 6 & 3 \\
\hline 33 & Evaluation & 20 & 15 & Principal Component Analysis & 11 & 9 & Virtual Reality & 7 & 3 & Patient Record & 6 & 5 \\
\hline 34 & MRI & 20 & 14 & GPU & 10 & 7 & $\mathrm{Xml}$ & 7 & 1 & Survival Analysis & 6 & 2 \\
\hline 35 & Teleradiology & 20 & 17 & Mammography & 10 & 8 & Hip Prosthesis & 6 & 1 & Anesthesia & 5 & 2 \\
\hline 36 & Knowledge-Based System & 19 & 13 & Mathematical Morphology & 10 & 7 & Magnetic Resonance Imaging & 6 & 4 & Clinical Protocols & 5 & 1 \\
\hline 37 & Monte Carlo & 19 & 12 & Matlab & 10 & 7 & Nonmem & 6 & 4 & Computer Program & 5 & 1 \\
\hline 38 & Computer Program & 18 & 7 & Medical Imaging & 10 & 8 & Pharmacodynamics & 6 & 6 & Correlated Responses & 5 & 5 \\
\hline 39 & Radiology & 18 & 18 & Monte Carlo Simulation & 10 & 5 & World Wide Web & 6 & 4 & Diabetes Mellitus & 5 & 3 \\
\hline 40 & Finite Element Method & 17 & 6 & Parkinson's Disease & 10 & 8 & 3D Reconstruction & 5 & 0 & Diagnosis & 5 & 3 \\
\hline
\end{tabular}

Abbreviations: Occ $=$ Occurrences; $\mathrm{Co}-\mathrm{oc}=$ Co-occurrence link strength. 


\section{Discussion}

CMPB is identified as a leading and core journal for biomedical researchers in the world. The journal is strongly connected to many other journals including IEEE Transactions on Biomedical Engineering and IEEE Transactions on Medical Imaging. The most cited CMPB paper is from the authors - Lihong Wang, Steven L. Jacques, Liqiong Zheng (published in 1995) receiving more than 2000 citations. Top CMPB authors include Yu-Chuan Li, James Geoffrey Chase and Geoffrey M. Chase and the top 3 institutions publishing in CMPB are Uppsala U (Sweden), Taipei Medical U (Taiwan) and INSERM (France). The journal is very diverse in terms of institutions and countries and is strongly influenced by North America and Europe. The highest number of CMPB papers were from the USA followed by the UK and Italy. In Asia, only China and Taiwan appear in the top 10 countries publishing in CMPB. Keyword co-occurrences analysis reveals an interesting co-relation between the biomedical area of research and methods that were used. Strong co-occurrences were identified for keywords such as classification, PACS, heart rate variability, survival analysis and simulation. In the last decade, keywords analysis found that topics relating to machine learning systems for variety of healthcare problems (including image processing and analysis) dominated other research fields in CMPB.

This bibliometric approach provides a practical review of the current leading trends of CMPB. However, it is worth noting that the result presented in this work consider publications until 31 December 2017. Therefore, note that in the future the results may evolve in different directions with new trends emerging in the journal. Additionally, the work follows the methodology and results available in the WoS core collection. Thus, any limitation that applies to this database, also applies to this study. Finally, each research field may have different results that may also condition the bibliometric results producing more publications or citations. In any case, the objective of this study is to identify the publication and citation trends of the journal in order to get a general overview of the current state of the art.

Following on from the bibliometric analysis, we now present some of the insights for the journal. There was a drop in number of papers published in 2015 in CMPB compared to the previous years for some reasons. Since then, the journal has consistently increased the 
number of publications. CMPB should try to attract quality papers consistently and a good and prompt review feedback will go a long way in achieving these objectives. There is an increasing number of papers published in last 10 years in CMPB which implies higher number of submissions and therefore higher manuscript handling workload for the Editor-inChief, editorial board members and reviewers. As such, effective and efficient review mechanism should be adopted to reduce this workload. Some of the strategies may include expanding the editorial board of the journal as well as rigorous initial screening of the manuscripts submitted to the journal for their suitability. CMPB should also encourage submissions involving (i) software tools along with its methodology application to a biomedical field (ii) state-of-the-art review to increase the citation appeal. It was found that the papers providing software tools and reviews are getting high citations compared to a regular paper proposing a methodology. The journal can expand its editorial board members to include authors of highly cited CMPB publications. It can attract quality submissions from their network and area of influence. CMPB should pay particular attention to submissions involving classification, segmentation, feature selection/ extraction, image processing as it has become central theme in the publications in last decade. In particular, submissions with deep learning methods should be encouraged further as it is emerging out to be a theme of considerable interest to the biomedical research community. In past, publications involving some type of computer simulation were popular but in recent decade publications with machine learning methods are more prominent. Some special issues by leading experts in the area could be regularly planned on the emerging topics of the journal such as deep learning applications in biomedical areas to reinvigorate quality and focussed submissions.

\section{Conclusions}

To celebrate the $50^{\text {th }}$ anniversary of CMPB, this paper presents a bibliometric analysis and overview of the major trends that have occurred in the journal in its publication history. The study uses the WoS core collection and the analysis was presented on all the papers published from the journal between 1970 and 2017. The trends suggest that CMPB is a world renowned publication outlet for biomedical researchers which has been growing in number of publications produced every year since 1970 . 
The study presented a range of analyses on the papers published and their citations to identify leading institutions, countries, authors, and key research areas. To better understand the bibliometric results, this study also presents the graphical and mapping analysis of the papers through the use of VOS viewer software. The study considered co-citation, bibliographic coupling, citation, co-authorship and co-occurrence of author keywords. These analyses can provide readers with better understanding and growing importance of CMPB journal.

\section{Acknowledgments:}

Authors acknowledge that they have not received any funding for carrying out the research. Authors also declare that there are not competing interests and ethics approval required for this study.

\section{References:}

[1] Van Eck, N.J., \& Waltman, L. (2010). Software survey: VOSviewer, a computer program for bibliometric mapping. Scientometrics, 84(2), 523-538.

[2] Kessler, M.M. (1963). Bibliographic coupling between scientific papers. American Documentation, 14(1), 10-25.

[3] Small, H. (1973). Co-citation in the scientific literature: A new measure of the relationship between two documents. Journal of the American Society for Information Science, 24, 265-269.

[4] Barley, S. R. (2016). 60 anniversary essay: Ruminations on how we became a mystery house and how we might get out. Administrative Science Quarterly, 61, 1-8.

[5] Dolgui (2018). Leading scholars in Production Research for the 55th volume anniversary of IJPR, International Journal of Production Research, 56 (1-2), 1-9.

[6] Van Fleet, D., Ray, D.F., Bedeian, A.G., Downey, H.K., Hunt, J.G., Griffin, R.W., Dalton, D., Vecchio, R.P., Kacmar, K.M., \& Feldman, D.C. (2006). The Journal of Management's first 30 years. Journal of Management, 32(4), 477-506.

[7] Meyer, R., \& Winer, R. S. (2014). Introduction to the JMR 50 anniversary special section. Journal of Marketing Research, 51, 83.

[8] Merigó, J.M., Mas-Tur, A., Roig-Tierno, N., \& Ribeiro-Soriano, D. (2015). A bibliometric overview of the Journal of Business Research between 1973 and 2014. Journal of Business Research, 68(12), 2645-2653.

[9] Cobo, M.J., Martínez, M.A., Gutiérrez-Salcedo, M., Fujita, H., \& Herrera-Viedma, E. (2015). 25 years at Knowledge-Based Systems: A bibliometric analysis. Knowledge-Based Systems, 80, 3-13. 
[10] Valenzuela, L., Merigó, J.M., Johnston, W., Nicolás, C. \& Jaramillo, F. (2017). Thirty years of the Journal of Business \& Industrial Marketing: A bibliometric analysis. Journal of Business \& Industrial Marketing, 32(1), 1-18.

[11] Merigó, J.M., Blanco-Mesa, F., Gil-Lafuente, A.M., \& Yager, R.R. (2017). Thirty years of the International Journal of Intelligent Systems: A bibliometric review. International Journal of Intelligent Systems, 32, 526-554.

[12] Broadus, R.N. (1987). Toward a definition of "Bibliometrics". Scientometrics, 12(56), 373-379.

[13] Pritchard, A. (1969). Statistical bibliography or bibliometrics? Journal of Documentation, 25, 348-349.

[14] Bar-llan, J. (2008). Informetrics at the beginning of the 21 century- a review. Journal of Informetrics, 2(1), 1-52.

[15] Podsakoff, P.M., MacKenzie, S.B., Podsakoff, N.P., \& Bachrach, D.G. (2008). Scholarly influence in the field of management: A bibliometric analysis of the determinants of university and author impact in the management literature in the past quarter century. Journal of Management, 34, 641-720.

[16] Bonilla, C., Merigó, J.M., \& Torres-Abad, C. (2015). Economics in Latin America: A bibliometric analysis, Scientometrics, 105(2), 1239-1252.

[17] Merigó, J.M., Cancino, C., Coronado, F., \& Urbano, D. (2016). Academic research in innovation: A country analysis. Scientometrics, 108, 559-593.

[18] Landström, H., Harirchi, G., \& Aström, F. (2012). Entrepreneurship: Exploring the knowledge base. Research Policy, 41, 1154-1181.

[19] Merigó, J.M., Gil-Lafuente, A.M., \& Yager, R.R. (2015). An overview of fuzzy research with bibliometrics indicators. Applied Soft Computing, 27, 420-433.

[20] Yu, D., Li, D.F., Merigó, J.M., \& Fang, L. (2016). Mapping development of linguistic decision making studies. Journal of Intelligent \& Fuzzy Systems, 30, 2727-2736.

[21] Blanco-Mesa, F., Merigó, J.M., \& Gil-Lafuente, A.M. (2017). Fuzzy decision making: A bibliometric-based review. Journal of Intelligent \& Fuzzy Systems, 32(3), 2033-2050.

[22] Wang, W., Laengle, S., Merigó, J.M., Yu, D., Herrera-Viedma, E., Cobo, M.J., \& Bouchon-Meunier, B. (2018). A bibliometric analysis of the first twenty-five years of the International Journal of Uncertainty, Fuzziness and Knowledge-Based Systems. International Journal of Uncertainty, Fuzziness and Knowledge-Based Systems, 26, 169-193.

[23] Cancino, C., Merigó, J.M., \& Coronado, F. (2017). Leading universities in innovation research, Journal of Innovation \& Knowledge, 2, 106-124.

[24] López-Herrera, A. G., Herrera-Viedma, E., Cobo, M. J., Martínez, M. A., Kou, G. \& Shi, Y. (2012). A conceptual snapshot of the first decade (2002-2011) of the International Journal of Information Technology \& Decision Making. International Journal of Information Technology \& Decision Making, 11, 247-270.

[25] Heck, J. L., Bremser, W. G. (1986). Six decades of The Accounting Review: A summary of author and institutional contributors. The Accounting Review, 61, 735744.

[26] Schwert, G.W. (1993). The Journal of Financial Economics: A retrospective evaluation (1974-91). Journal of Financial Economics, 33(3), 369-424. 
[27] Ramos-Rodríguez, A.R., \& Ruiz-Navarro, J. (2004). Changes in the intellectual structure of strategic management research: A bibliometric study of the Strategic Management Journal, 1980-2000. Strategic Management Journal, 25, 981-1004.

[28] García-Merino, M.T., Pereira-do-Carmo, M.L., \& Santos-Álvarez, M.V. (2006). 25 years of Technovation: Characterisation and evolution of the journal. Technovation, 26(12), 1303-1316.

[29] Biemans, W., Griffin, A., \& Moenaert, R. (2007). Twenty Years of the Journal of Product Innovation Management: History, Participants, and Knowledge Stock and Flows. Journal of Product Innovation Management, 24(3), 193-213.

[30] Vosner, H.B., Kokol, P., Bobek, S., Zeleznik, D., \& Zavrsnik, J. (2016). A bibliometric retrospective of the Journal Computers in Human Behavior, Computers in Human Behavior, 65, 46-58.

[31] Laengle, S., Merigó, J.M., Miranda, J., Slowinski, R., Bomze, I., Borgonovo, E., Dyson, R.G., Oliveira, J.F., \& Teunter, R. (2017). Forty years of the European Journal of Operational Research: A bibliometric overview. European Journal of Operational Research, 262(3), 803-816.

[32] Cancino, C., Merigó, J.M., Coronado, F., Dessouky, Y., \& Dessouky, M. (2017b). Forty years of Computers \& Industrial Engineering: A bibliometric analysis. Computers \& Industrial Engineering, 113, 614-629.

[33] Merigó, J.M., Pedrycz, W., Weber, R., \& de la Sotta, C. (2018). Fifty years of Information Sciences: A bibliometric overview. Information Sciences, 432, 245-268.

[34] Martínez-López, F.J., Merigó, J.M., Valenzuela, L., \& Nicolás, C. (2018). Fifty years of the European Journal of Marketing: A bibliometric analysis. European Journal of Marketing, 52(1-2), 439-468.

[35] Yu, D., Xu, Z.S., Kao, Y., \& Lin, C.T. (2018). The Structure and Citation Landscape of IEEE Transactions on Fuzzy Systems (1994-2015). IEEE Transactions on Fuzzy Systems, 26, 430-442.

[36] Gaviria-Marín, M., Merigó, J.M., Popa, S. (2018). Twenty years of the Journal of Knowledge Management: A bibliometric analysis. Journal of Knowledge Management, 22(8), 1655-1687.

[37] Ding, Y., Rousseau, R., \& Wolfram, D. (2014). Measuring scholarly impact: Methods and practice, Springer, Switzerland.

[38] Mulet-Forteza, C., Martorell-Cunill, O., Merigó, J.M., Genovart-Balaguer, J., \& Mauleon-Mendez, E. (2018). Journal of Travel \& Tourism Marketing: A bibliometric analysis. Journal of Travel \& Tourism Marketing, 35(9), 1201-1221.

[39] Tur-Porcar, A., Mas-Tur, A., Merigó, J.M., Roig-Tierno, N., \& Watt, J. (2018). A bibliometric history of the Journal of Psychology between 1936 and 2015. Journal of Psychology, 152, 199-225.

[40] Hirsch, J.E. (2005). An index to quantify an individual's scientific research output. Proceedings of the National Academy of Sciences of the United States of America, 102(46), 16569-16572.

[41] Laengle, S., Modak, N.M., Merigó, J.M., \& de la Sotta, C. (2018). Thirty years of the International Journal of Computer Integrated Manufacturing: A bibliometric analysis. International Journal of Computer Integrated Manufacturing, 31(12), 1247-1268.

[42] Laengle, S., Modak, N.M., Merigó, J.M., Zurita, G. (2018). Twenty-five years of Group Decision and Negotiation: A bibliometric overview. Group Decision and Negotiation, 27(4), 505-542. 
[43] Alonso, S., Cabrerizo, F.J., Herrera-Viedma, E., \& Herrera, F. (2009). H-index: A review focused on its variants, computation, and standarization for different scientific fields. Journal of Informetrics, 3(4), 273-289.

[44] Cobo, M.J., Lopez-Herrera, A.G., Herrera-Viedma, E., \& Herrera, F. (2011). Science mapping software tools: Review, analysis and cooperative study among tools. Journal of the American Society for Information Science and Technology, 62(7), 1382-1402.

[45] Baier-Fuentes, H., Cascon-Katchadourian, J., Martinez, M.A., Herrera-Viedma, E., \& Merigó, J.M. (2018). A bibliometric overview of the International Journal of Interactive Multimedia and Artificial Intelligence. International Journal of Interactive Multimedia and Artificial Intelligence, 19(5), 9-16.

[46] Tang, M., \& Liao, H. C., \& Su, S. F. (2018). A bibliometric overview and visualization of the International Journal of Fuzzy Systems between 2007 and 2017. International Journal of Fuzzy Systems, 20, 1403-1422.

[47] Faust, O., 2018. Documenting and predicting topic changes in Computers in Biology and Medicine: A bibliometric keyword analysis from 1990 to 2017. Informatics in Medicine Unlocked, 11, pp.15-27 